Fraile Jurado, P., Fernández Díaz, M. (2018): "Escala, esfuerzo digitalizador y fractalidad en la línea de costa”, GeoFocus (Articulos), $n^{\circ} 21$, p. 253-277. ISSN: 1578-5157 http://dx.doi.org/10.21138/GF.568

\title{
ESCALA, ESFUERZO DIGITALIZADOR Y FRACTALIDAD EN LA LÍNEA DE COSTA
}

\author{
PABLO FRAILE JURADO ${ }^{1}$, MIGUEL FERNÁNDEZ DÍAZ ${ }^{1}$ \\ ${ }^{1}$ Departamento de Geografía Física y A.G.R., Universidad de Sevilla \\ C/ María de Padilla s/n, 41004, Sevilla \\ pfraile@us.es
}

\begin{abstract}
RESUMEN
En este trabajo se analizan las interrelaciones entre escala, esfuerzo digitalizador y dimensión fractal en los procesos de digitalización de diferentes tipos de línea de costa en siete sectores costeros diferentes. A partir de ortoimágenes con una resolución espacial de 0,5 metros, se digitalizaron 25 tramos costeros diferentes a cinco escalas distintas, de 1:10.000 a 1:1.500, identificando la longitud de cada uno de los tramos obtenidos y el número de vértices. Se calculó la dimensión fractal de cada uno de los tramos digitalizados a cada escala, analizando esta variable con la longitud y el número de vértices. Los resultados indican que algunos tramos costeros de gran complejidad (marismas y costas rocosas) presentan una estructura fractal. En estos casos es posible predecir la longitud total de línea de costa a gran escala a partir de la longitud de líneas de costa a menor escala.
\end{abstract}

Palabras clave: línea de costa, longitud, vectorial, fractal.

\section{SCALE, DIGITIZING EFFORT AND FRACTALITY OF THE SHORELINE}

\section{ABSTRACT}

The aim of this work is to analyse the interrelations between scale, digitizing effort and fractal dimension in the digitization processes of different types of shoreline in seven coastal sectors. From orthoimages with a spatial resolution of 0.5 meters, 25 different coastal segments were digitized at five different scales, ranging from 1: 10,000 to 1: 1,500. The length of each of the obtained segments and their number of vertices were calculated. The fractal dimension of each of the digitized sections was calculated at each scale, analyzing this variable with the length and number of vertices. The results show that some very complex coastal sectors (tidal marshes and rocky coasts) have a fractal structure. In these cases it is possible to predict the total length of largescale coastline from the length obtained digitizing at a smaller scale.

Keywords: shoreline, length, vector, fractal. 
Fraile Jurado, P., Fernández Díaz, M. (2018): "Escala, esfuerzo digitalizador y fractalidad en la línea de costa”, GeoFocus (Articulos), $n^{\circ} 21$, p. 253-277. ISSN: 1578-5157 http://dx.doi.org/10.21138/GF.568

\section{Introducción}

Durante las dos últimas décadas se ha vivido un proceso acelerado de digitalización de la información geográfica. La evolución desde las fuentes tradicionales de información (mapas, fotografías aéreas) hacia el uso de elementos vectoriales y ráster ha sido cada vez más común, especialmente desde la generalización de los procesos de compartición online, como los impulsados por la directiva Inspire. La necesidad de representación de una amplísima cantidad de modelos, terrenos y objetos geográficos hace que no siempre sea tan simple la reducción a los clásicos puntos, líneas y polígonos. En el caso del presente análisis la línea, un elemento aparentemente perfecto para la reproducción de la costa se convierte en sí misma en un objeto problemático y cuya delimitación se aleja mucho de la exactitud dada la propia naturaleza fractal de la costa, así como su progresividad en cuanto a su delimitación (López-Martín y Fraile-Jurado, 2015).

La línea de costa es, por definición, el objeto geográfico de mayor dinamismo, puesto que consiste en la zona de contacto entre la superficie emergida y la superficie marina, esta última en constante movimiento horizontal y vertical, causado por fenómenos de diferentes escalas espaciales y temporales: oleaje, mareas, viento, presión atmosférica, cambio climático y subida del nivel del mar (Fraile, 2011). Asimismo, la superficie emergida también sufre notables modificaciones a diferentes escalas temporales: desde movimientos verticales de velocidades medidas en $\mathrm{mm} / \mathrm{año}$, por subida del nivel del mar, por tectónica... (Peltier y Tushingham, 1991) a súbitos cambios causados por los efectos de temporales y el oleaje en general.

El objetivo de este trabajo es valorar y evaluar las interrelaciones entre los principales parámetros que definen los procesos de digitalización de diferentes tipos de línea de costa: escala, longitud de la línea, coste de la digitalización y dimensión fractal. Los resultados de este trabajo deben aportar información crítica a la hora de decidir la escala óptima de digitalización, permitiendo economizar tiempo y esfuerzos en este tipo de procesos que suelen conllevar largos periodos de tiempo, en ocasiones innecesarios. Tras esta introducción, el trabajo consta en el apartado 2, de una revisión bibliográfica en la que se aborda el estado de la cuestión, en el apartado 3 el área de estudio, datos y métodos, mientras que los resultados y discusión están incluidos en los apartados 4 y 5 respectivamente. En el anexo se frecen tres tablas con la totalidad de los resultados obtenidos.

\section{Revisión bibliográfica}

La digitalización de la línea de costa obliga a un proceso de toma de decisiones que si bien es común para cualquier otro tipo de objetivo, se torna crítico en este caso: qué criterios de digitalización tomar, y qué escala de digitalización elegir. Sobre los criterios de digitalización se han realizado numerosas aportaciones (Casal et al. 2010, Zhang et al. 2002, Ojeda, 2000; Dolan et al. 2000), de las que se puede extraer la importancia de determinar el propósito de la digitalización a la hora de identificar qué se considera línea de costa y qué no. Sobre la cuestión de la escala óptima de digitalización, los trabajos realizados resultan significativamente más erráticos sin que se haya identificado una escala aceptable de trabajo. En algunos casos, se han empleado escalas desmesuradamente grandes, sin que se haya considerado ni las características de la imagen que servía como referencia ni las características del objeto, en este caso el tipo de costa, a digitalizar. En 
Fraile Jurado, P., Fernández Díaz, M. (2018): "Escala, esfuerzo digitalizador y fractalidad en la línea de costa", GeoFocus (Articulos), $n^{\circ}$ 21, p. 253-277. ISSN: 1578-5157 http://dx.doi.org/10.21138/GF.568

el trabajo de Casal et al. (2010), pese a definir unos claros criterios de digitalización, se empleó una única escala de 1:700, independientemente de que tanto el tamaño de celdilla ( 0,5 metros) como las formas de algunos tramos rectilíneos, se puedan considerar inadecuados para dicha escala, por exceso.

Asimismo, es bastante frecuente identificar en la literatura sobre costas mediciones de líneas costeras en las que, sin indicar la escala de digitalización, se identifican medidas que en comparación con otras publicaciones resultan cortas. Tal es el caso de la línea de costa de España peninsular, que de acuerdo con Terán et al. (1985) mide $3.144 \mathrm{~km}, 5.849 \mathrm{~km}$ según el INE en su informe de 1985 , y $4.872 \mathrm{~km}$ al consultar en esta misma fuente en 2017 , sin que se indique en ninguno de estos trabajos ni la escala de digitalización ni la precisión de la fuente. La importancia de una adecuada digitalización de la línea de costa ha sido destacada por Crowell et al. (1999) al condicionar no solo los cálculos derivados de esta como los relacionados con la erosión costera, sino incluso decisiones administrativas y urbanísticas, derivadas del trazado de la línea de costa o de las tasas de erosión registradas, tal y como es frecuente en Estados Unidos. El propio concepto de línea de costa, como interfaz física entre superficie emergida y marina, implica profundas asunciones conceptuales a la hora de medir o digitalizar procesos sobre esta, tal y como ha sido subrayado por Boak y Turner (2005).

La relación entre análisis fractal y longitud de línea de costa es sobradamente conocida, incluso en la cultura popular, puesto que el trabajo de Benoit Mandelbrot (1967) que da inicio a esta rama de las matemáticas se tituló ¿Cuánto mide la línea de costa de Gran Bretaña? (How long is the coast of Britain?). El principio más difundido de este trabajo se resume en la cita textual del autor: La evidencia empírica sugiere una regla que, si se extrapola, muestra que la longitud se incrementa sin límite a medida que la longitud del tramo disminuye.

No obstante, parece preciso clarificar el concepto estricto de fractal para contextualizar el marco de estudio del presente trabajo. Los fractales, del latín fractum, quebrado, son formas de geometría no euclidiana caracterizadas por cierta autosimilitud dentro de una continua irregularidad. El concepto de fractal, por tanto, alude a un tipo de objeto cuya estructura tiende a repetirse independientemente del tramo en el que se observe e incluso de la escala de observación. Para cuantificar cuán fractal es un objeto se suele emplear el concepto de dimensión fractal, es decir, cuánto llena un objeto (el fractal) un espacio conforme se amplía hacia escalas de mayor detalle. Al medir la dimensión fractal o fractalidad de un objeto, se obtiene un valor que frecuentemente tiende a repetirse independientemente de la escala a la que se mida, aunque no se trata de un valor único ni estable. Existen numerosas aproximaciones para medir este parámetro, en función del método empleado. Los más comunes, especialmente por su facilidad para ser implementados de manera manual en la época del desarrollo inicial de esta rama del análisis geométrico, tienen como base común el recuento de objetos idénticos entre sí (círculos, cuadrados, así como otras figuras más complejas) de un tamaño cada vez menor. La proporción entre el número de objetos existentes en un área determinada y el tamaño de dicho objeto es la base para el cálculo de diferentes indicadores de dimensión fractal (Mandelbrot, 1967, 1977).

Otros conceptos cercanos a las matemáticas fractales son los de autoafinidad y autosimilitud, siendo el primero mucho más frecuente en el estudio de la línea de costa que el segundo. La autosimilitud es la propiedad por la cual un objeto mantiene su forma 
Fraile Jurado, P., Fernández Díaz, M. (2018): "Escala, esfuerzo digitalizador y fractalidad en la línea de costa", GeoFocus (Articulos), $n^{\circ}$ 21, p. 253-277. ISSN: 1578-5157 http://dx.doi.org/10.21138/GF.568

independientemente de la escala de observación, mientras que la autoafinidad es la propiedad de un objeto por la cual este cambia su forma al modificarse la escala de observación, manteniéndose la dimensión fractal sin cambios.

La geometría tradicional se ha utilizado para el estudio de las geoformas del paisajes (muy comúnmente en geomorfología fluvial) mediante la simplificación introducida para la descripción de figuras geométricas. Sin embargo, las matemáticas fractales se han aplicado a la descripción de formas irregulares que pueden ser caracterizadas por un patrón y un número real (Ángeles et al. 2004).

Durante las últimas décadas, varios autores han usado las propiedades fractales de la línea de costa para describir objetos y fenómenos costeros. Algunos trabajos se han centrado en la caracterización de la línea de costa, mientras este mismo elemento también ha sido empleado, a semejanza de Mandelbrot, para testar metodologías orientadas al cálculo de la dimensión fractal de diferentes tipos de objetos. La diversidad de tipologías de trabajos que han empleado aproximaciones fractales al análisis de objetos costeros es tan amplia como las propias matemáticas fractales. De entre dichas tipologías cabe destacar el trabajo de Schwimmer (2008), en el que se emplea el cálculo de la dimensión fractal para la identificación de procesos erosivos en marismas, permitiendo este análisis eludir los problemas a la disponibilidades de series temporales de imágenes y sobre todo del momento de marea asociado a la fotografía; el uso de los fractales para la identificación de estructuras costeras, como los trabajos publicados por Sharma y Byrne (2010) para identificar líneas de costa en Titán; así como los análisis fractales para la determinación de las estructuras de estuarios y marismas, y de redes fluviales (Snow, 1989; Ángeles et al. 2004).

\section{3. Área de estudio, datos y métodos}

\section{1. Área de estudio}

Para este trabajo se eligieron siete sectores costeros diferentes situados en la costa andaluza (figura 1). Esta costa andaluza presenta una amplia diversidad de morfología de líneas de costa, condicionadas tanto por diferentes factores hidrodinámicos como geológicos. Hidrodinámicamente, se trata de una costa bañada por dos masas de agua de características muy diferentes. La costa bañada por el Océano Atlántico presenta un régimen mesomareal (con máximos de hasta 3,4 metros) en la provincia de Huelva y parte de la de Cádiz. Desde las marismas de Sancti-Petri hasta el Estrecho de Gibraltar se produce una disminución del rango mareal, que en el área del Estrecho alcanza rangos no superiores a 1 metro, y que disminuye en la provincia de Málaga hasta máximo de 0,6 m, configurando el Estrecho como zona de transición en este aspecto. En las áreas atlánticas de mayor rango mareal es posible identificar amplias zonas de marismas mareales de gran desarrollo, que prácticamente desaparecen en las zonas de rangos mareales intermedios y mínimos. La costa atlántica andaluza presenta amplias playas de carácter esencialmente rectilíneo, condicionadas tanto por las características morfo-estructurales de la costa andaluza (Ojeda, 2000) como por la existencia de importantes fuentes de sedimentos arenosos. La costa mediterránea presenta amplios sectores rocosos y acantilados de rango medio y amplio, debido a la presencia de los Sistemas Béticos, que condicionan tanto la existencia de fuertes pendientes como la ausencia de una plataforma continental desarrollada que permitiese disipar la energía del oleaje incidente. En el 
Fraile Jurado, P., Fernández Díaz, M. (2018): "Escala, esfuerzo digitalizador y fractalidad en la línea de costa”, GeoFocus (Articulos), $n^{\circ} 21$, p. 253-277. ISSN: 1578-5157 http://dx.doi.org/10.21138/GF.568

área mediterránea, así mismo, es frecuente la presencia de infraestructuras portuarias y de protección de playas, como espigones.

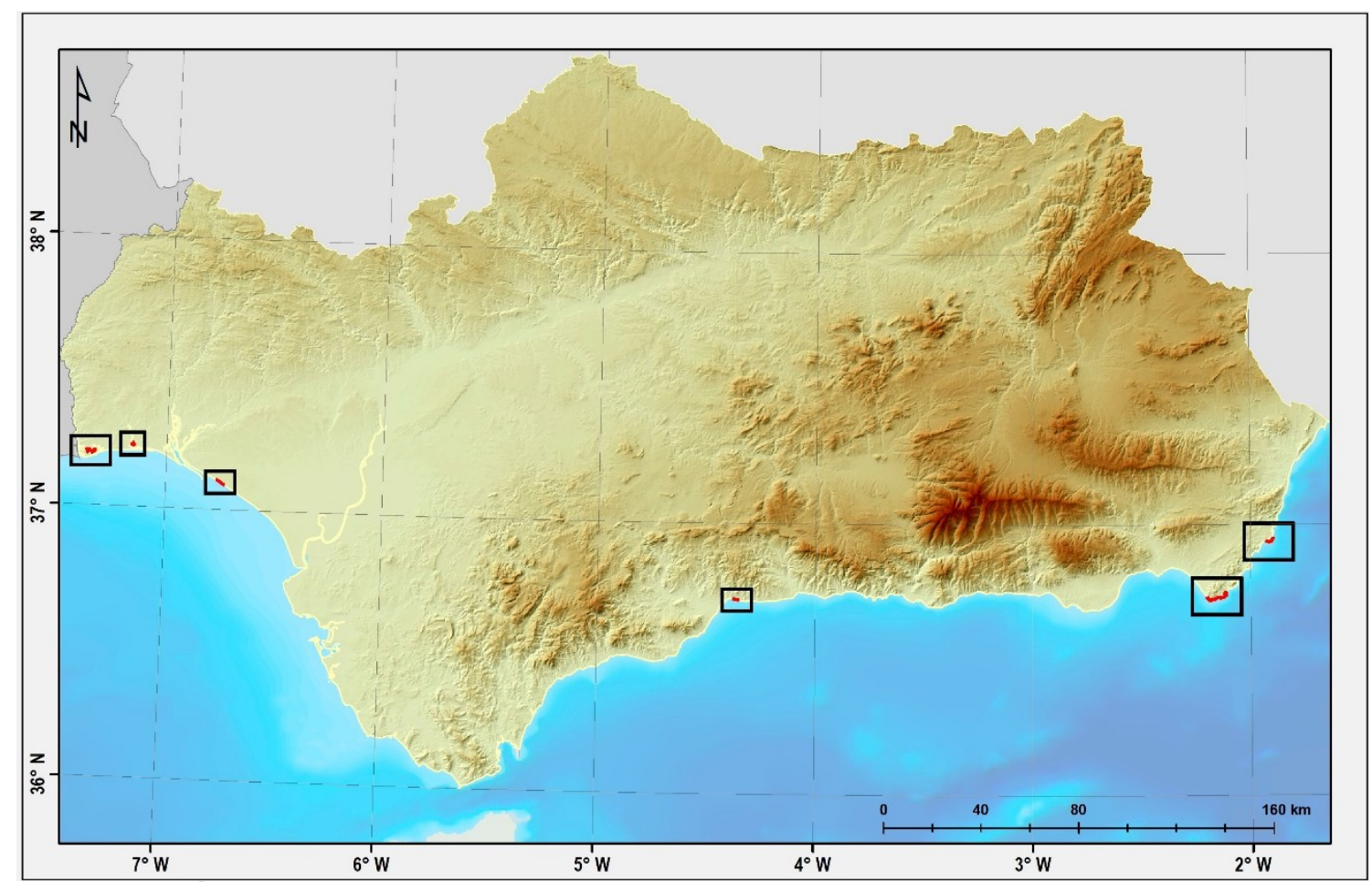

Figura 1. Área de estudio. En los recuadros negros, con línea roja, aparecen los sectores costeros digitalizados. En el segundo marco empezando por el oeste se encuentran dos de las áreas de estudio, las del río Carreras y las del entorno de Isla Cristina.

Con la intención de caracterizar diferentes morfologías, con distinto grado de intervención humana, se eligieron 7 áreas de estudio, que esencialmente cuatro tipos de costa diferentes:

a) Sectores de marisma: marismas de Isla Cristina (figura 2), marismas del río Carreras (figura 3), y marismas del río Piedras (figura 4).

b) Sectores acantilados o accidentados: costas del Cabo de Gata (figura 5) y del entorno de Carboneras (figura 6).

c) Playas abiertas: playa de Mazagón (figura 7).

d) Sectores con fuerte intervención humana en la forma de la línea de costa: áreas de espigones en Málaga (figura 8). 
Fraile Jurado, P., Fernández Díaz, M. (2018): "Escala, esfuerzo digitalizador y fractalidad en la línea de costa", GeoFocus (Articulos), $n^{\circ} 21$, p. 253-277. ISSN: 1578-5157 http://dx.doi.org/10.21138/GF.568

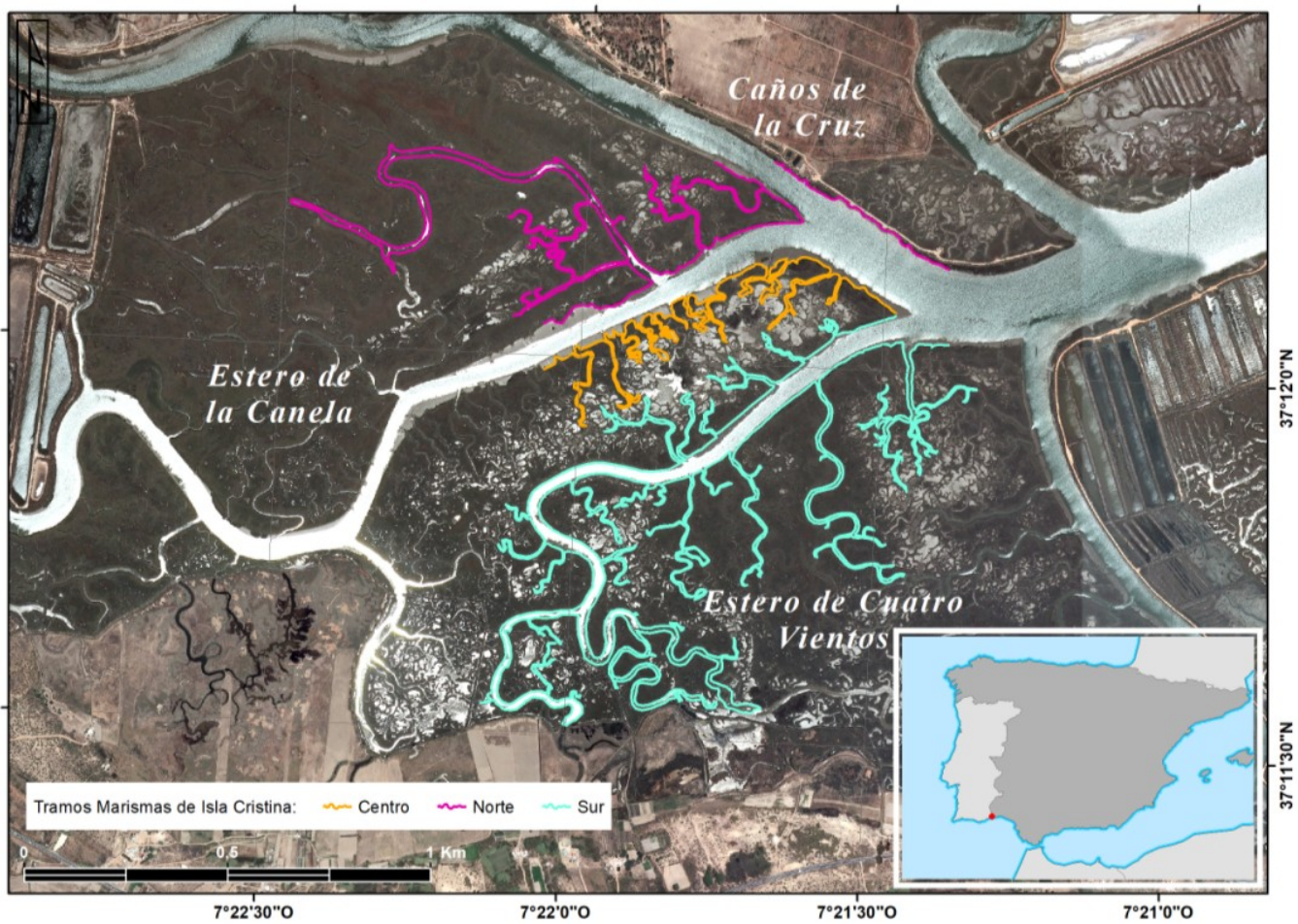

Figura 2. Sector de las marismas del entorno de Isla Cristina.

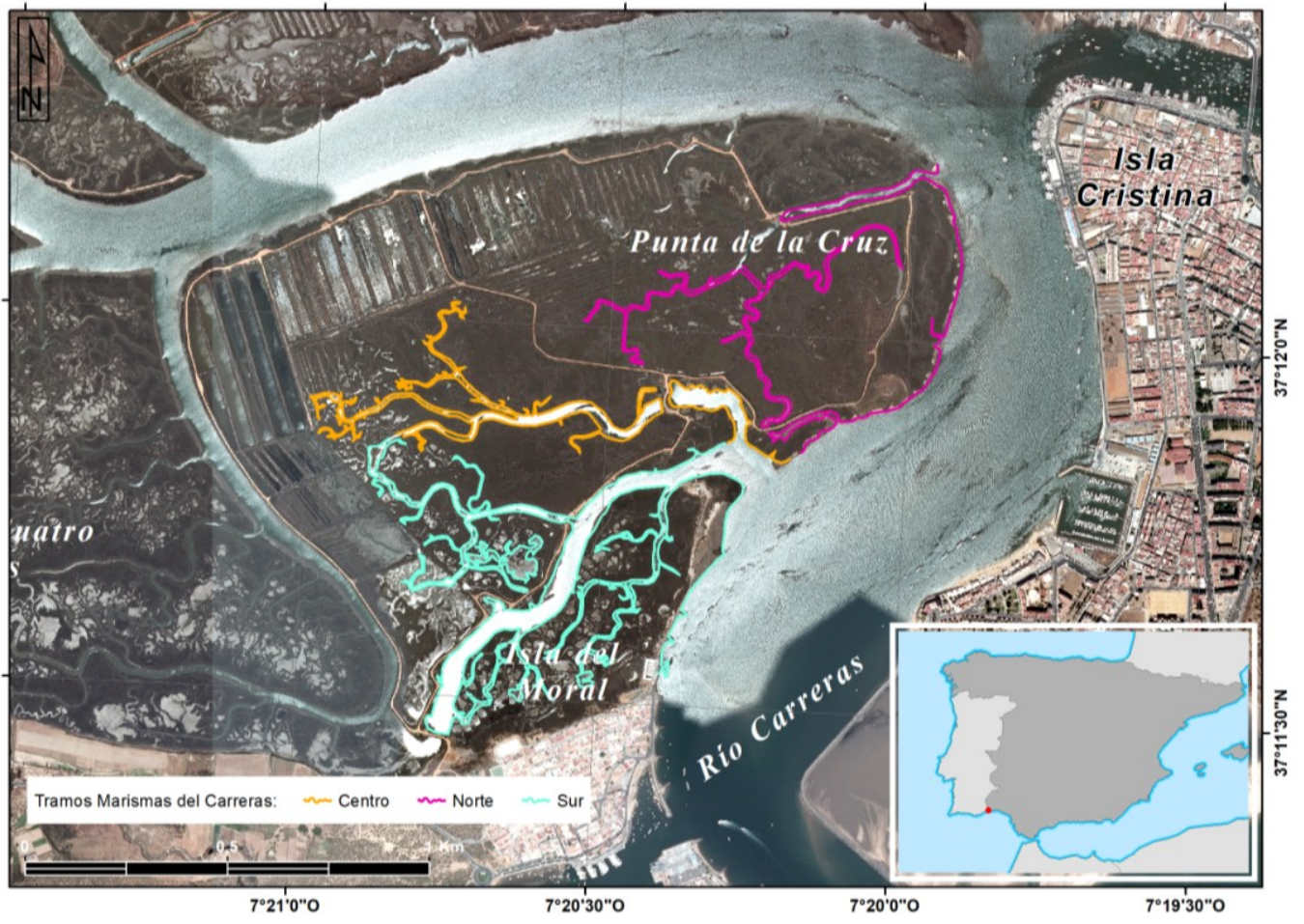

Figura 3. Sector de las marismas del río Carreras. 
Fraile Jurado, P., Fernández Díaz, M. (2018): "Escala, esfuerzo digitalizador y fractalidad en la línea de costa", GeoFocus (Articulos), $n^{\circ} 21$, p. 253-277. ISSN: 1578-5157 http://dx.doi.org/10.21138/GF.568

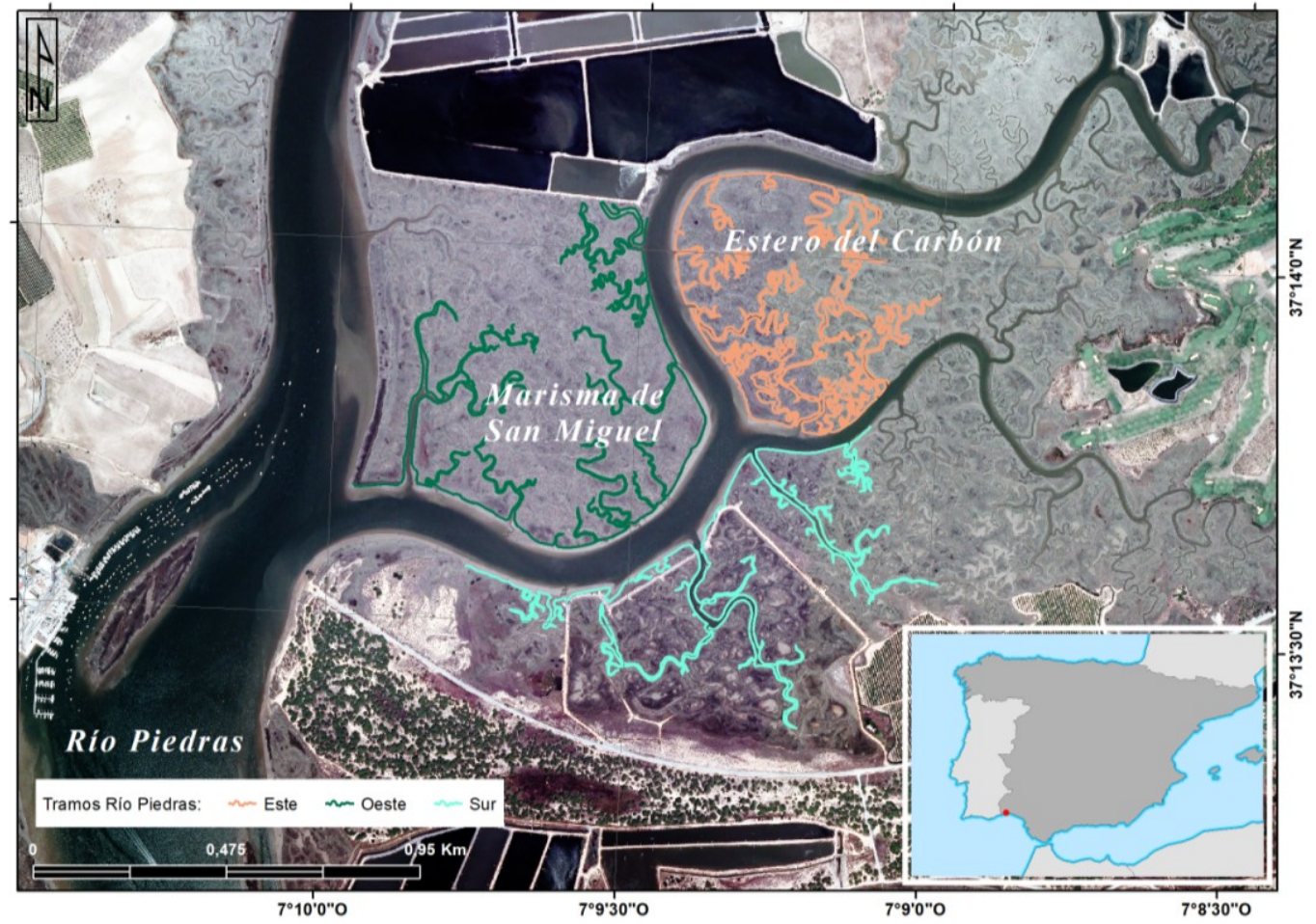

Figura 4. Sector de las marismas del río Piedras.

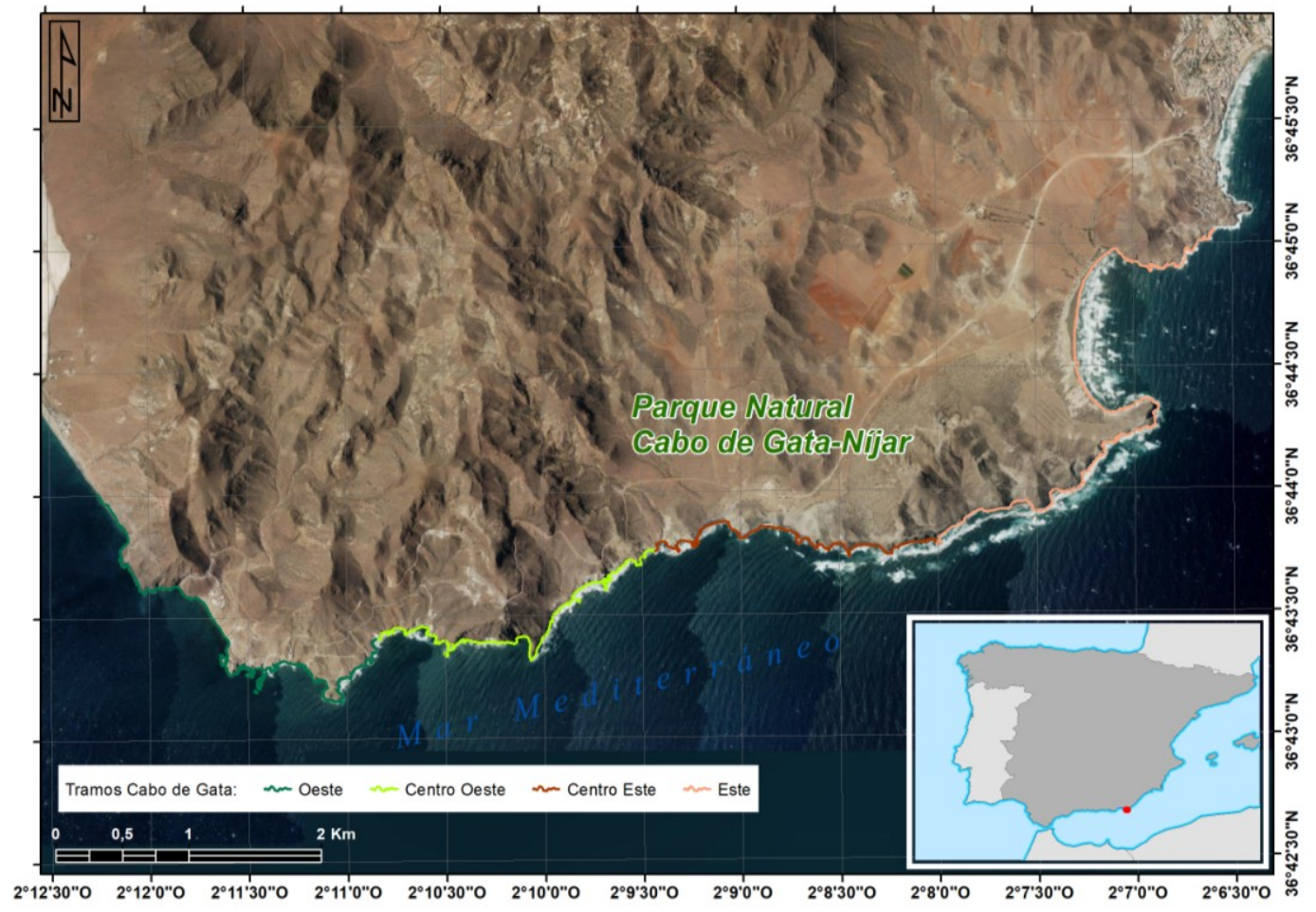

Figura 5. Sector de la costa del Cabo de Gata. 
Fraile Jurado, P., Fernández Díaz, M. (2018): "Escala, esfuerzo digitalizador y fractalidad en la línea de costa", GeoFocus (Articulos), $n^{\circ}$ 21, p. 253-277. ISSN: 1578-5157 http://dx.doi.org/10.21138/GF.568

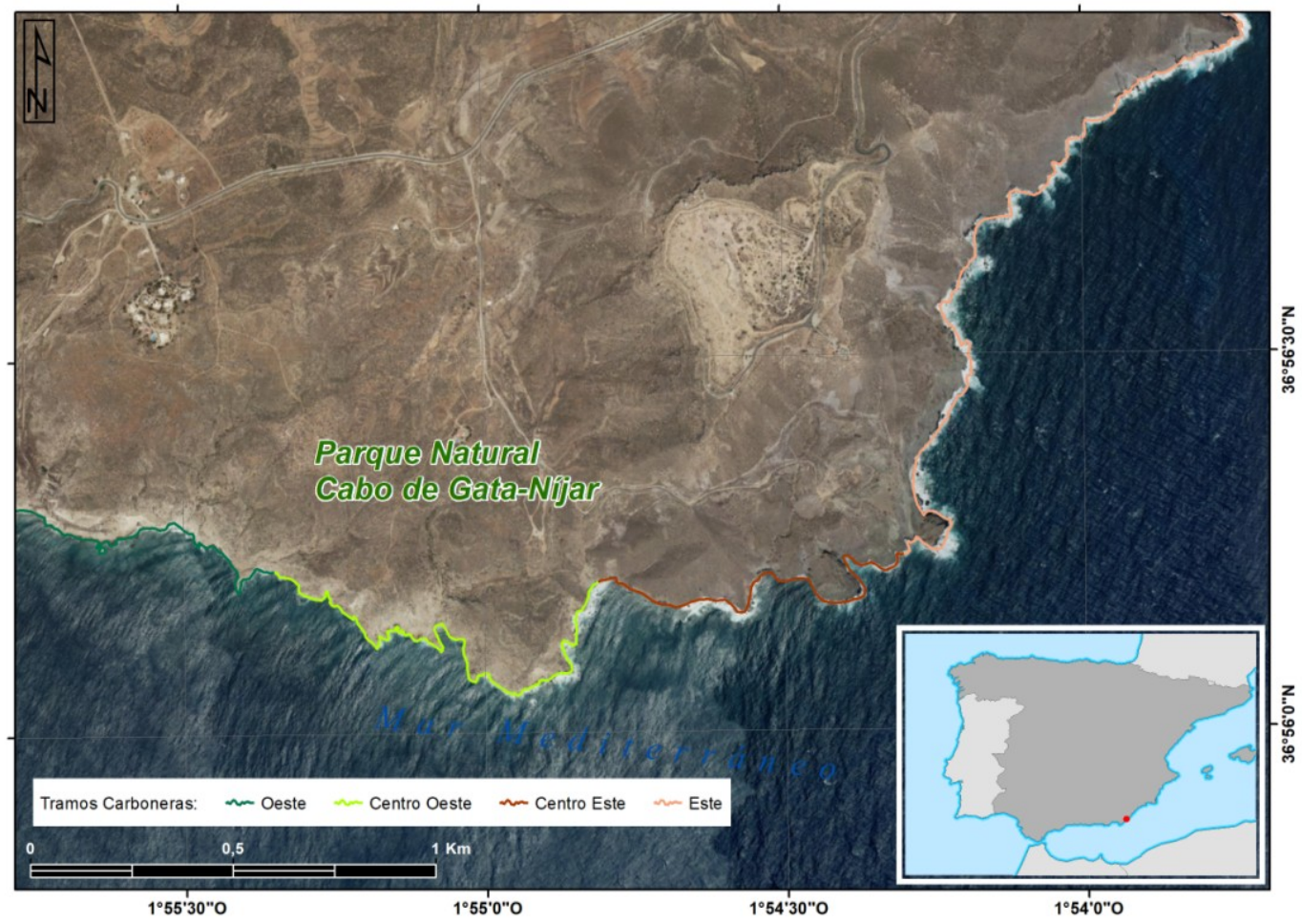

Figura 6. Sector de la costa del entorno de Carboneras

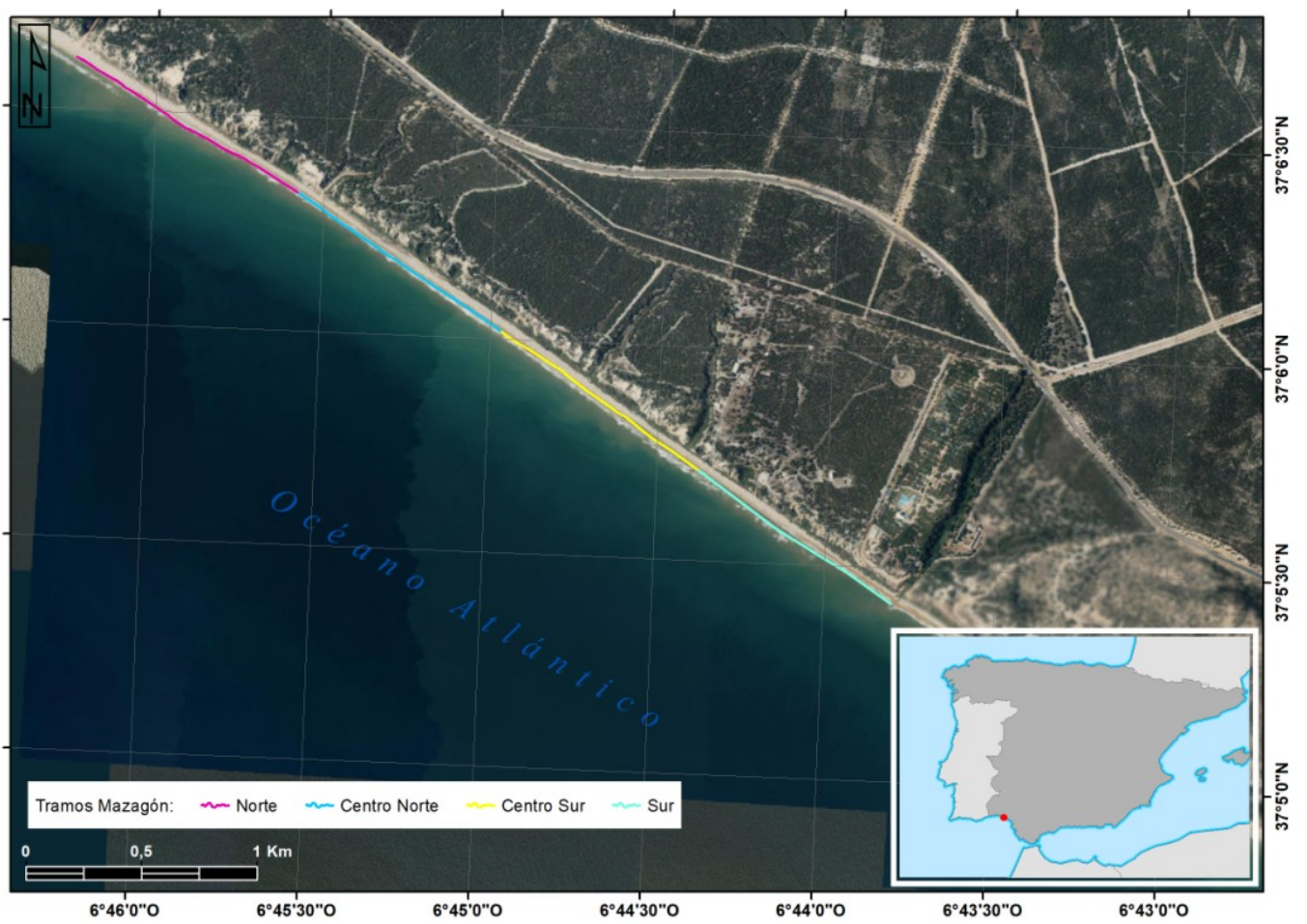

Figura 7. Sector de la costa de Mazagón 
Fraile Jurado, P., Fernández Díaz, M. (2018): "Escala, esfuerzo digitalizador y fractalidad en la línea de costa", GeoFocus (Articulos), $n^{\circ} 21$, p. 253-277. ISSN: 1578-5157 http://dx.doi.org/10.21138/GF.568

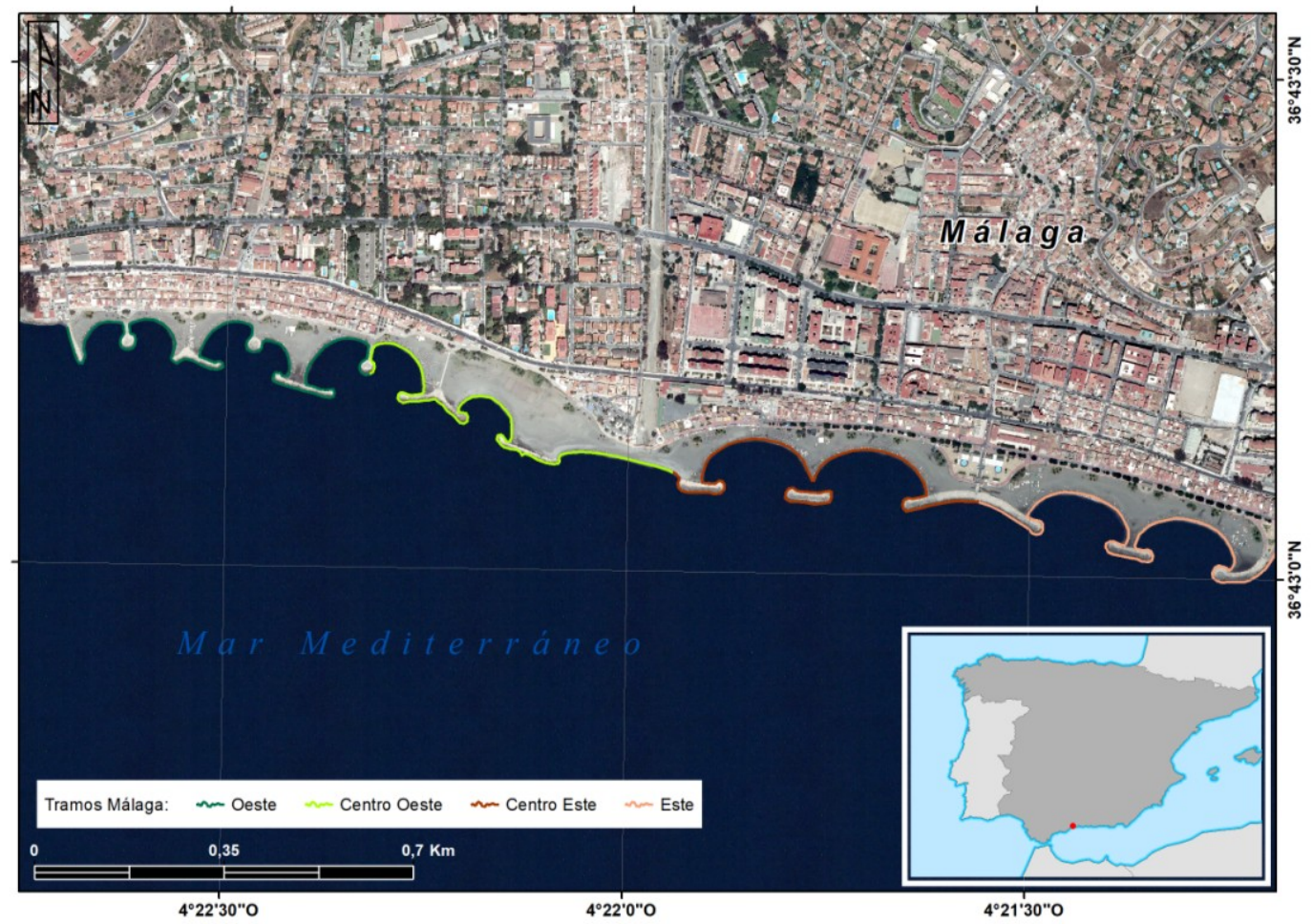

Figura 8. Sector de la costa de Málaga.

\subsection{Datos empleados}

Para los procesos de digitalización se empleó la Ortofoto del Cuadrante NW con resolución de $0,5 \mathrm{~m}$ generada a partir de un vuelo fotogramétrico del año 2011, disponible en cuatro canales (RGB e infrarrojo), de los cuales se empleó la combinación de color natural. Esta ortofoto fue proporcionada por la Consejería de Agricultura, Pesca y Medio Ambiente de la Junta de Andalucía.

Se eligió esa imagen debido a tres motivos esenciales: i) una resolución espacial de suficiente detalle $(0,5$ metros por celdilla) como para permitir digitalizaciones de detalle, ii) que la fotografía, en las áreas de estudio elegidas que cuentan con rango mesomareal, está tomada en una situación de bajamar, por lo que en las áreas de marisma, los caños mareales se observan vacíos y su delimitación resulta más sencilla y precisa en las planicies intermareales de las áreas de mayor rango mareal, y iii) el hecho de que pese a la resolución, óptima para escalas de detalle, los elementos digitalizados son correctamente visualizados a escalas intermedias, sin que se observen ruidos ni distorsiones en la imagen.

\subsection{Métodos}

El proceso metodológico desarrollado fue el siguiente: 
Fraile Jurado, P., Fernández Díaz, M. (2018): "Escala, esfuerzo digitalizador y fractalidad en la línea de costa”, GeoFocus (Artículos), $n^{\circ} 21$, p. 253-277. ISSN: 1578-5157 http://dx.doi.org/10.21138/GF.568

1. Se identificó la escala máxima de digitalización, que fue estimada en 1:1.500, de acuerdo con un análisis visual de la resolución de la imagen.

2. Se delimitaron entre tres y cuatro tramos diferentes dentro de cada sector, con la intención de facilitar los análisis estadísticos realizados, asegurando la homogeneidad de los mismos (figuras 2 a 7). Al nombre de cada tramo se le añadió $\mathrm{O}, \mathrm{CO}, \mathrm{CE}$ y E en función de si se situaban en la parte oeste, centro-oeste, centro-este o este de cada área de estudio. Del mismo modo, a las áreas de estudio con orientación predominante norte - sur se les añadió N, CN, CS y CS para identificar los tramos localizados al norte, centro norte, centro sur y sur del área de estudio.

3. A continuación, se digitalizaron las líneas de costa de los sectores costeros recogidos en el apartado "área de estudio" a la escala máxima de digitalización 1:1.500, así como a las escalas $1: 2.500,1: 5.000,1: 7.500$ y 1:10.000, empleado la herramienta de zoom del software ArcGIS 10.3 sobre una misma y única imagen. Los criterios de digitalización fueron los siguientes (figura 9):

a. En las costas arenosas se siguió la línea de vegetación permanente, límite comúnmente atribuido en la bibliografía al máximo nivel del mar alcanzado durante un ciclo anual en condiciones normales.

b. En las costas acantiladas se distinguió entre dos tipos de línea de costa: acantiladas "a plomo", en las que se digitalizó el límite roca / agua, y las costas acantiladas de pendiente media, en las que se empleó la última marca húmeda como límite (Casal el at. 2010) de las rocas conectadas a la superficie continental, excluyendo rocas exentas o farallones.

c. En las infraestructuras costeras y portuarias, que suelen caracterizarse por su verticalidad, se siguió el mismo criterio que en las costas acantiladas a plomo.

d. En las marismas se siguió la marca de vegetación, debido a que se trata del límite entre la zona inundada por la pleamar y la no inundada comúnmente (Casal et al. 2012). El criterio general de digitalización, en cualquier caso, pretende identificar el límite entre la superficie emergida y las aguas marinas, considerándose por tanto en como costa cualquier límite natural entre vegetación permanente y estas aguas en el caso de las marismas.

4. Se calculó la longitud y número de vértices de cada uno de los objetos generados.

5. Con la intención de poder realizar análisis comparados se recalcularon longitud y número de vértices en base 1 tomando como referencia el valor de cada tramo a escala 1:10.000.

6. Se calculó la fractalidad de todas las líneas de costa resultantes mediante el uso del software libre Fractalyse. La fractalidad de la línea de costa fue calculada a partir de dos métodos tipo boxcounting. Este método consiste en la generación sucesiva de diferentes mallas, de tal manera que se cubra la totalidad del contorno de cualquier objeto con el menor número de cuadrados posible. La proporción entre el número de cuadrados necesarios y el lado del cuadrado se relacionan la siguiente ecuación:

$$
\operatorname{Ln} N(r)=D * \operatorname{Ln}(1 / r)+C
$$


Fraile Jurado, P., Fernández Díaz, M. (2018): "Escala, esfuerzo digitalizador y fractalidad en la línea de costa”, GeoFocus (Articulos), $n^{\circ} 21$, p. 253-277. ISSN: 1578-5157 http://dx.doi.org/10.21138/GF.568

siendo $\mathrm{N}=$ Número de cuadrados necesarios para cubrir una figura, $\mathrm{D}=$ Pendiente de una recta de regresión lineal con $\operatorname{Ln} \mathrm{N}(\mathrm{r})$ en el eje y y $\operatorname{Ln}(1 / \mathrm{r})$ en el eje $\mathrm{x}, \mathrm{r}=$ Lado de cada cuadrado, y $\mathrm{C}=$ Constante en la recta de regresión lineal.

7. Se realizó un análisis de correlaciones y de regresión lineal con la intención de caracterizar las relaciones entre las variables esfuerzo digitalizador (número de vértices), precisión de la línea (longitud de la línea de costa) y dimensión fractal. Para poder homogeneizar las variables longitud de la línea de costa y número de vértices, se estandarizaron en base 1 empleando como valor de referencia el registro correspondiente a la escala 1:10.000.

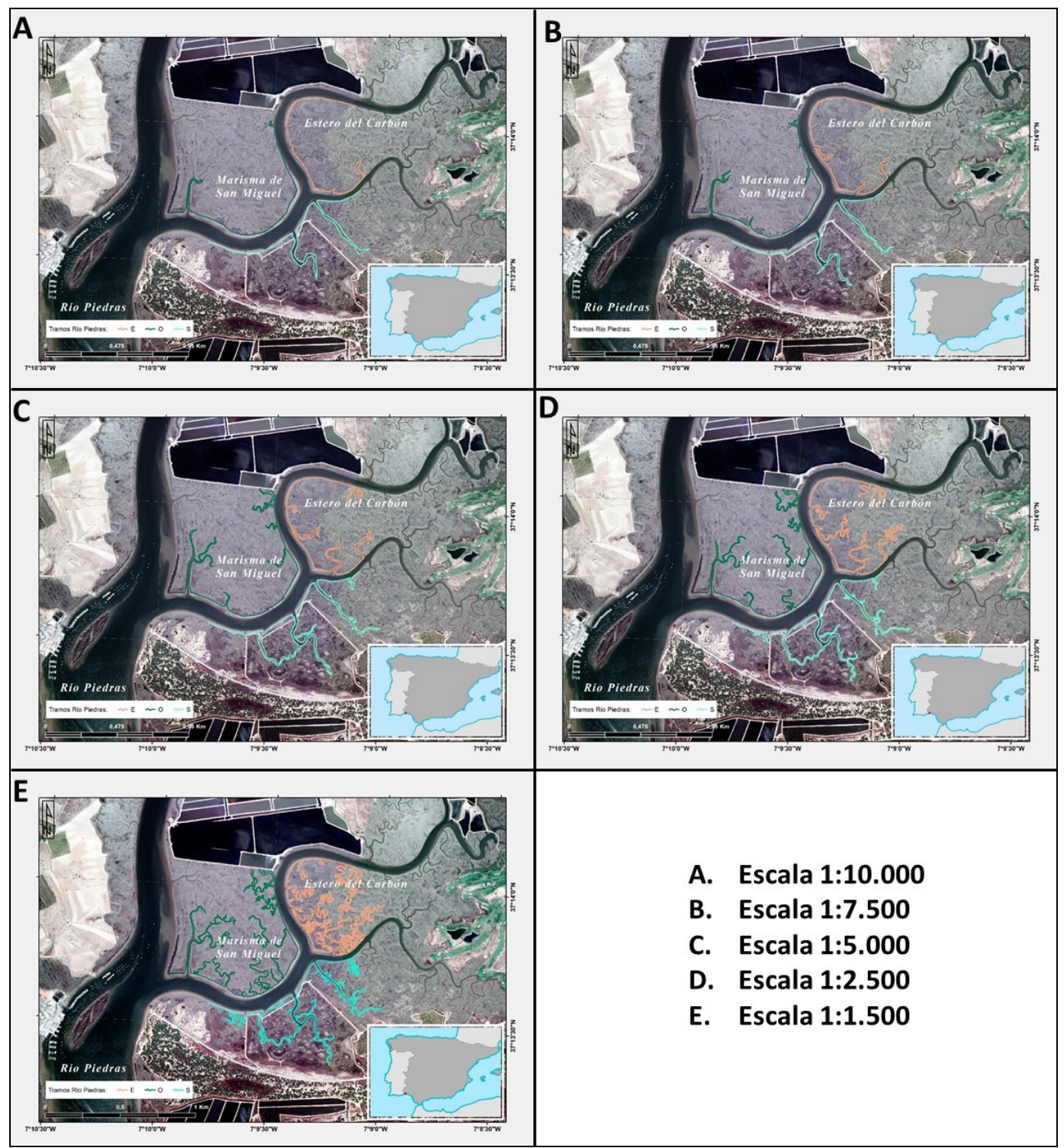

Figura 9. Digitalización de las marismas del río Piedras a distintas escalas.

(C) Los autores www.geofocus.org 
Fraile Jurado, P., Fernández Díaz, M. (2018): "Escala, esfuerzo digitalizador y fractalidad en la línea de costa”, GeoFocus (Articulos), $n^{\circ}$ 21, p. 253-277. ISSN: 1578-5157 http://dx.doi.org/10.21138/GF.568

\section{Descripción y análisis de resultados}

Los resultados de este trabajo son muy extensos, por lo que se han estructurado en cuatro subapartados en los que se exponen los resultados obtenidos del análisis de relaciones entre esfuerzo digitalizador y longitud de la línea de costa, de autocorrelación, entre fractalidad y longitud de la línea de costa, y entre fractalidad y esfuerzo digitalizador. Los valores obtenidos sobre la longitud de cada tramo, número de vértices y fractalidad, base para el análisis, se hallan recogidos en las tablas 1,2 y 3 del apéndice.

\subsection{Esfuerzo digitalizador vs precisión de la línea de costa}

Los resultados obtenidos muestran que existen diferentes tipos de relación entre el esfuerzo digitalizador y la precisión del resultado obtenido. Esta relación, tal y como se muestra en la figura 10 es un indicador del tamaño medio de cada segmento digitalizado en las diferentes escalas. La precisión de la línea de costa (es decir, el tamaño de los segmentos) únicamente se incrementa en una dirección. Los resultados indican que existen determinados tipos de línea de costa cuya longitud se incrementa mucho más en función de la escala que el resto, tal y como se observa en la figura 10 En este sentido, existen dos modelos opuestos entre los tipos de costa analizados, y una gran variedad de situaciones intermedias en las que se sitúan el resto de tipos de costa. Las costas arenosas expuestas, como el tramo analizado en Mazagón, presentan una relación muy baja entre el incremento del esfuerzo digitalizador y la precisión de la línea de costa. La situación opuesta está representada por las costas de marismas mareales, en las que se identifica un incremento de la longitud -precisión- de la línea de costa que presenta una relación no lineal (exponencial), de tal modo que cuanto más se incrementa el esfuerzo digitalizador, mayor es el incremento de la longitud costera.

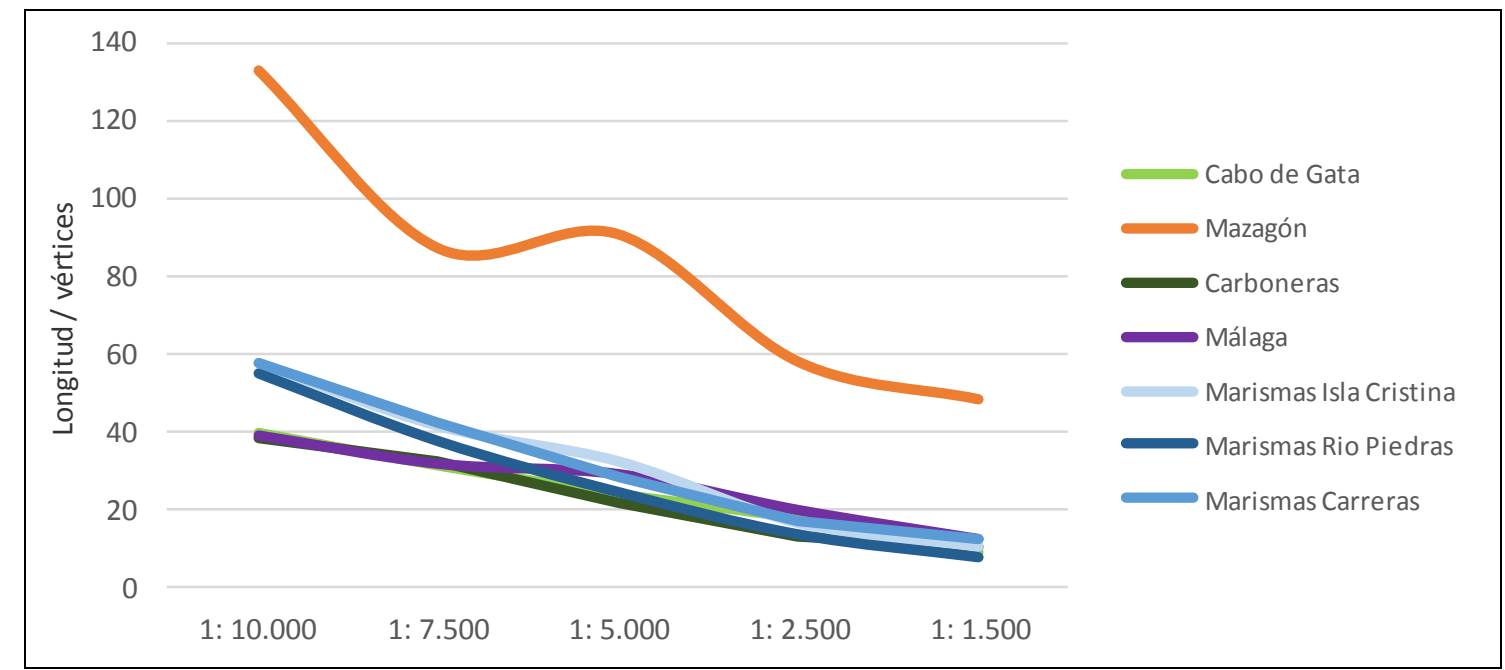

Figura 10. Relación entre el tamaño medio del segmento (longitud $/ \mathrm{n}^{0}$ vértices)(en metros) y la escala de digitalización. 
Fraile Jurado, P., Fernández Díaz, M. (2018): "Escala, esfuerzo digitalizador y fractalidad en la línea de costa”, GeoFocus (Articulos), $n^{\circ} 21$, p. 253-277. ISSN: 1578-5157 http://dx.doi.org/10.21138/GF.568

No obstante, para valorar adecuadamente los resultados obtenidos, es preciso considerar la limitación del tamaño de la pantalla, especialmente relevante en los tramos de costas rectilíneas, donde el autor de la digitalización suele identificar vértices aparentemente innecesarios en tramos rectilíneos de la línea de costa, debido a las limitaciones introducidas por el tamaño y resolución de la pantalla empleada para digitalizar. Ante una pantalla de dimensiones infinitas, el número de vértices sería aún más bajo, por lo que la línea de costa de la playa (la naranja en la figura 10) se mantendría próxima a la horizontalidad.

En el caso de las marismas objeto de estudio (figura 11), es evidente que existen una intensa relación entre el esfuerzo digitalizador y la precisión (entendida como longitud resultante) de la línea de costa. Pese a que tanto el modelo cuadrático como el lineal explican correctamente la varianza obtenida, el modelo cuadrático parece ser adecuarse ligeramente mejor a los resultados obtenidos.

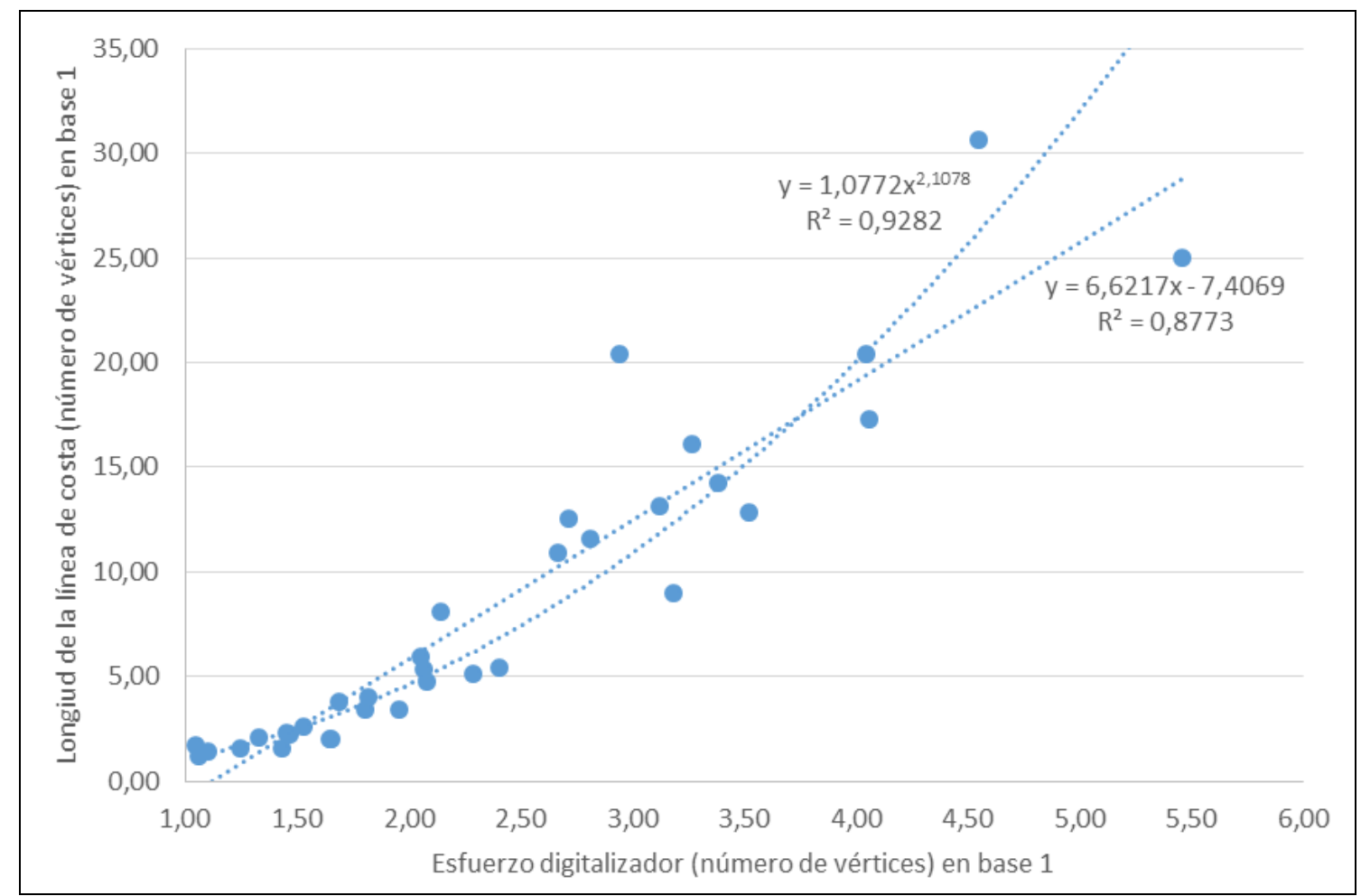

Figura 11. Relación entre el esfuerzo digitalizador y la longitud de la línea de costa en las marismas en todas las escalas abordadas en el trabajo. Los datos están en base 1 , a partir de los registros medidos a escala 1:10.000. 
Fraile Jurado, P., Fernández Díaz, M. (2018): "Escala, esfuerzo digitalizador y fractalidad en la línea de costa”, GeoFocus (Articulos), $n^{\circ} 21$, p. 253-277. ISSN: 1578-5157 http://dx.doi.org/10.21138/GF.568

\subsection{Autocorrelación de la longitud de la línea de costa}

En el análisis de autocorrelación de todos los registros con la escala inmediatamente superior se obtuvieron los resultados de la tabla 4. Si bien los resultados obtenidos muestran coeficientes de correlación de Pearson altos, únicamente en 14 de los tramos analizados este valor resultó estadísticamente significativo, debido al pequeño tamaño de cada una de las muestras. En cualquier caso, se identificó una elevada autocorrelación escalar en las costas más complejas, tanto en las marismas como en las costas del Cabo de Gata (figura 12).

La identificación de esta elevada autocorrelación implica la posibilidad de predecir la longitud obtenida en la línea de costa a partir de escalas de escaso detalle como 1:10.000 o 1:7.500.

Tabla 1. Coeficientes de correlación de Pearson para el análisis de autocorrelación de la longitud de la línea de costa en cada tramo estudiado. Los valores significativos se identifican mediante un asterisco (*).

\begin{tabular}{|l|c|}
\hline Marismas del Carreras_S & $0,998^{*}$ \\
\hline Río Piedras_E & $0,993^{*}$ \\
\hline Río Piedras_O & $0,987^{*}$ \\
\hline Málaga_CO & $0,977^{*}$ \\
\hline Río Piedras_S & $0,968^{*}$ \\
\hline Marismas de Isla Cristina_S & $0,966^{*}$ \\
\hline Cabo de Gata_CO & $0,899^{*}$ \\
\hline Marismas de Isla Cristina_C & $0,882^{*}$ \\
\hline Marismas del Carreras_N & $0,882^{*}$ \\
\hline Marismas del Carreras_C & $0,824^{*}$ \\
\hline Carboneras_O & $0,808^{*}$ \\
\hline Cabo de Gata_O & $0,794^{*}$ \\
\hline Málaga_E & $0,775^{*}$ \\
\hline
\end{tabular}

\begin{tabular}{|l|c|}
\hline Marismas de Isla Cristina_N & $0,760^{*}$ \\
\hline Cabo de Gata_CE & 0,655 \\
\hline Carboneras_E & 0,574 \\
\hline Cabo de Gata_E & 0,553 \\
\hline Málaga_CE & 0,407 \\
\hline Carboneras_CE & $-0,011$ \\
\hline Mazagón_S & $-0,28$ \\
\hline Carboneras_CO & $-0,311$ \\
\hline Mazagón_CS & $-0,628$ \\
\hline Mazagón_CN & $-0,749^{*}$ \\
\hline Mazagón_N & $-0,757^{*}$ \\
\hline Málaga_O & $-0,967^{*}$ \\
\hline
\end{tabular}


Fraile Jurado, P., Fernández Díaz, M. (2018): “Escala, esfuerzo digitalizador y fractalidad en la línea de costa”, GeoFocus (Articulos), $n^{\circ} 21$, p. 253-277. ISSN: 1578-5157 http://dx.doi.org/10.21138/GF.568

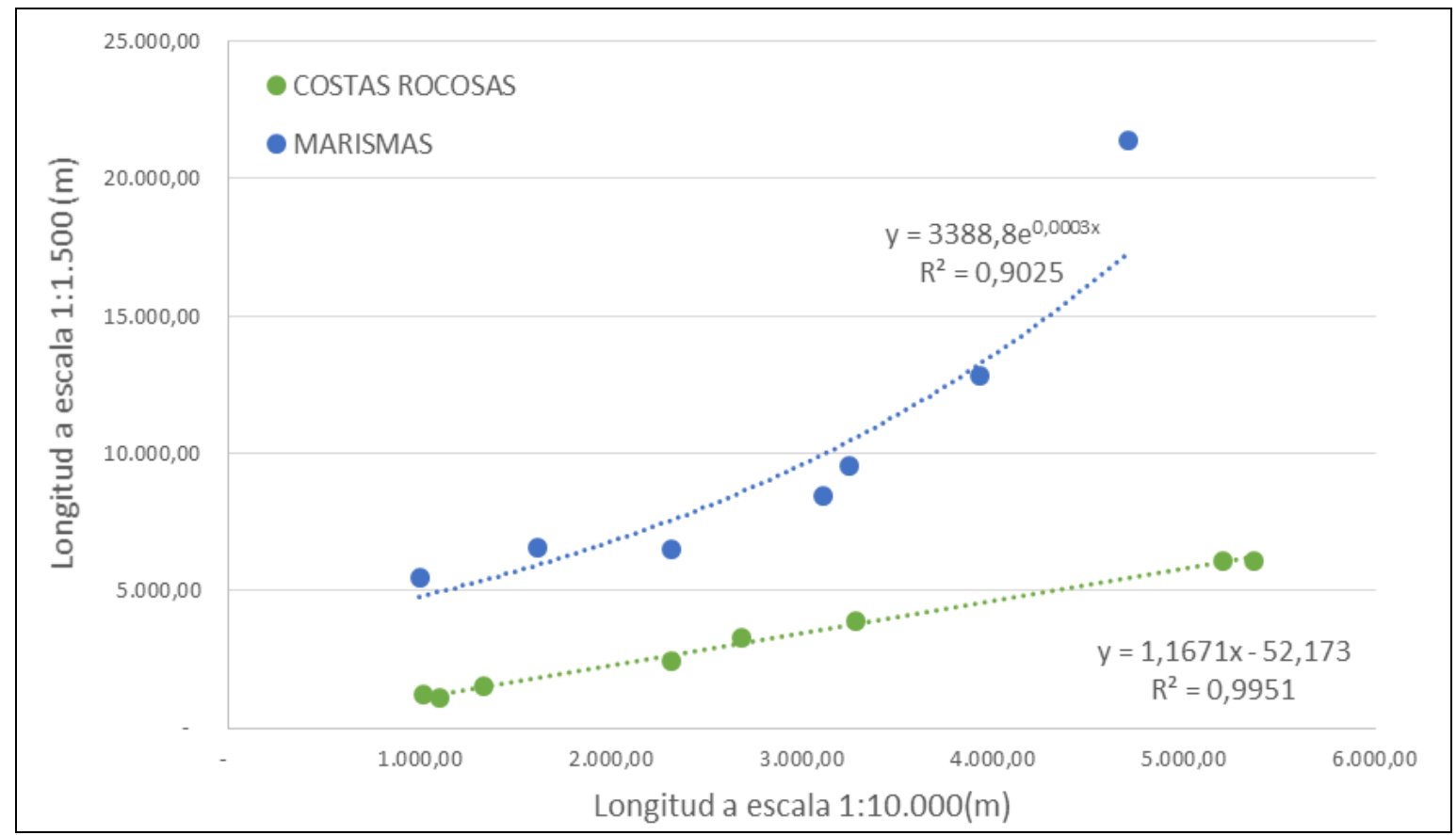

Figura 12. Autocorrelación entre la longitud de la línea de costa a escala 1:10.000 y a escala 1:1.500 para costas rocosas y marismas.

\subsection{Dimensión fractal vs longitud de la línea de costa}

La existencia de relación entre dimensión fractal y longitud de la línea de costa únicamente se identificó en los sectores analizados en Cabo de Gata y en las marismas del río Piedras (figuras 13 y 14), mientras que en los demás la relación no fue significativa.

La interpretación de estos resultados implica que en las costas más rugosas o de mayor complejidad (acantilados y marismas) la dimensión fractal se incrementa en relación a la escala, mientras que en zonas costeras con menor rugosidad (las playas como principal ejemplo) no existe dicha relación. Por ello, la dimensión fractal puede considerarse un elemento predictivo de la longitud total de un sector costero únicamente si este es muy rugoso. 
Fraile Jurado, P., Fernández Díaz, M. (2018): "Escala, esfuerzo digitalizador y fractalidad en la línea de costa”, GeoFocus (Articulos), $n^{\circ} 21$, p. 253-277. ISSN: 1578-5157 http://dx.doi.org/10.21138/GF.568

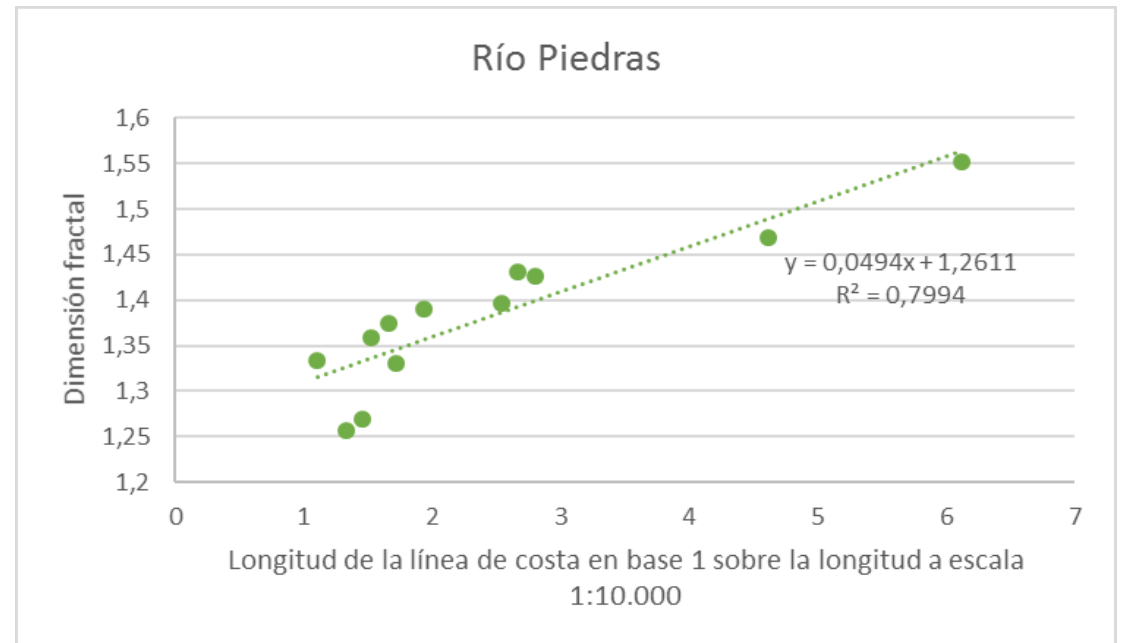

Figura 13. Análisis de regresión lineal simple entre la dimensión fractal (eje y) y la longitud de la línea de costa (eje x). Río Piedras.

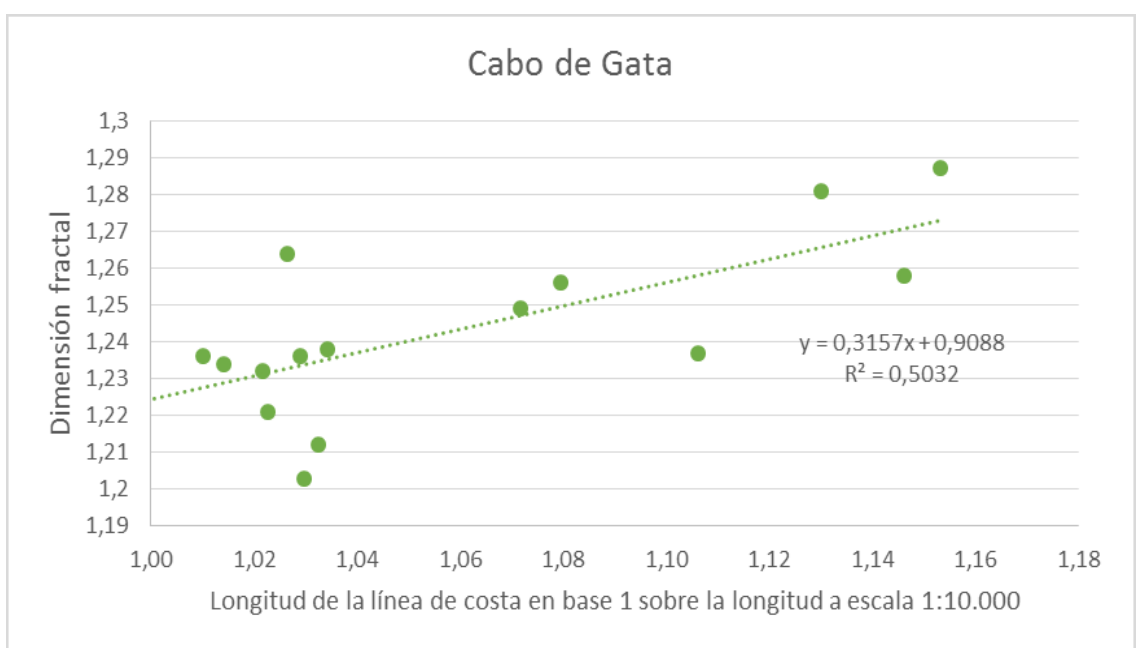

Figura 14. Análisis de regresión lineal simple entre la dimensión fractal (eje y) y la longitud de la línea de costa (eje x). Cabo de Gata

\subsection{Esfuerzo digitalizador vs dimensión fractal}

La relación entre el esfuerzo digitalizador y la dimensión fractal se ha identificado como significativa en las costas de Cabo de Gata (figura 17), de Carboneras, y en las marismas de Isla Cristina y del Río Piedras, mientras que en el resto de áreas de estudio no se ha evidenciado ninguna relación. En el caso de las marismas (figuras 15 y 16), el análisis mediante una relación logarítmica muestra un coeficiente de correlación de Pearson significativamente más alto que mediante la clásica relación lineal, como los que aparecen recogidos en la tabla 5. 
Fraile Jurado, P., Fernández Díaz, M. (2018): "Escala, esfuerzo digitalizador y fractalidad en la línea de costa”, GeoFocus (Articulos), $n^{\circ} 21$, p. 253-277. ISSN: 1578-5157 http://dx.doi.org/10.21138/GF.568

Tabla 2. Valor del coeficiente de correlación de Pearson (r) entre las variables número de vértices y dimensión fractal en el conjunto de los tramos de cada sector.

\begin{tabular}{|l|c|}
\cline { 2 - 2 } \multicolumn{1}{c|}{} & \multicolumn{1}{c|}{ r } \\
\hline Marismas del Río Piedras & 0,839 \\
\hline Marismas de Isla Cristina & 0,774 \\
\hline Cabo de Gata & 0,629 \\
\hline Carboneras & 0,451 \\
\hline Marismas del Carreras & 0,222 \\
\hline Málaga & 0,025 \\
\hline Mazagón & $-0,193$ \\
\hline
\end{tabular}

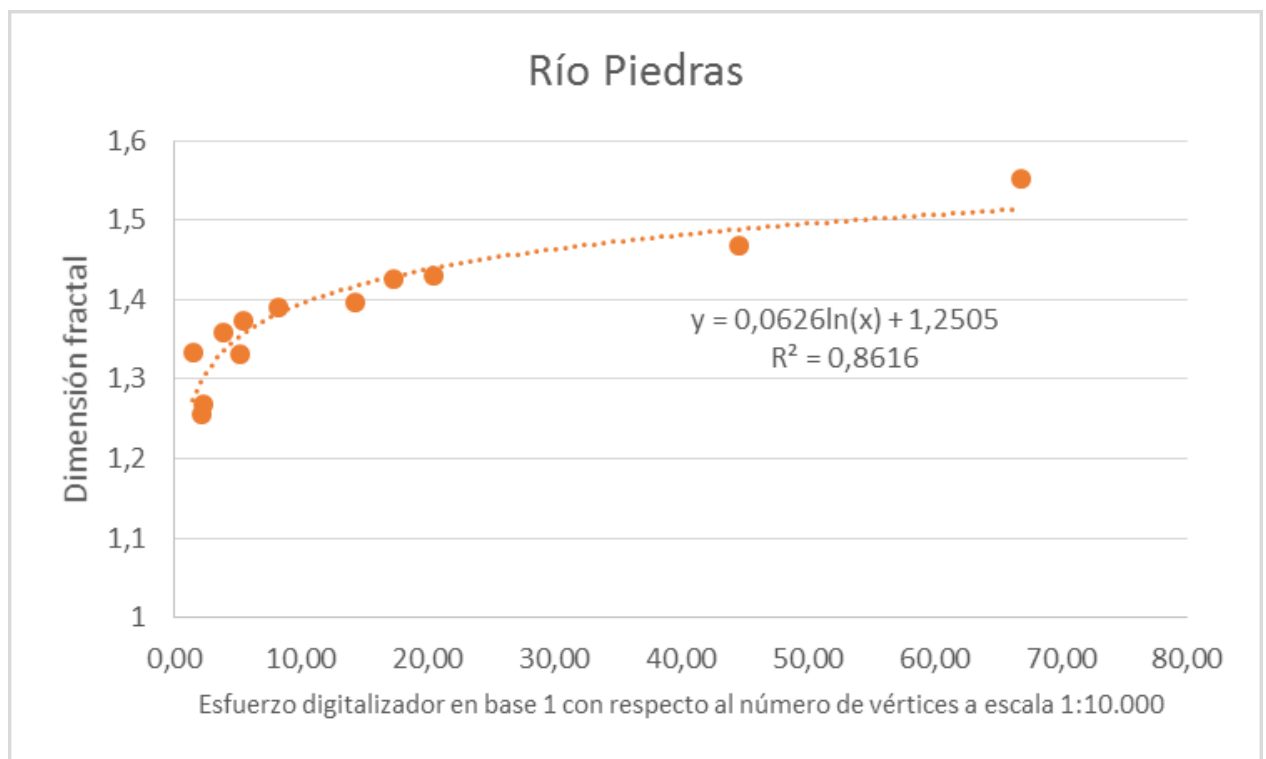

Figura 15. Análisis de regresión mediante modelo logarítmico entre las variables número de vértices (eje $\mathrm{x}$ ) y dimensión fractal (eje y) para el sector de las marismas del río Piedras. 
Fraile Jurado, P., Fernández Díaz, M. (2018): “Escala, esfuerzo digitalizador y fractalidad en la línea de costa”, GeoFocus (Articulos), $n^{\circ}$ 21, p. 253-277. ISSN: 1578-5157 http://dx.doi.org/10.21138/GF.568

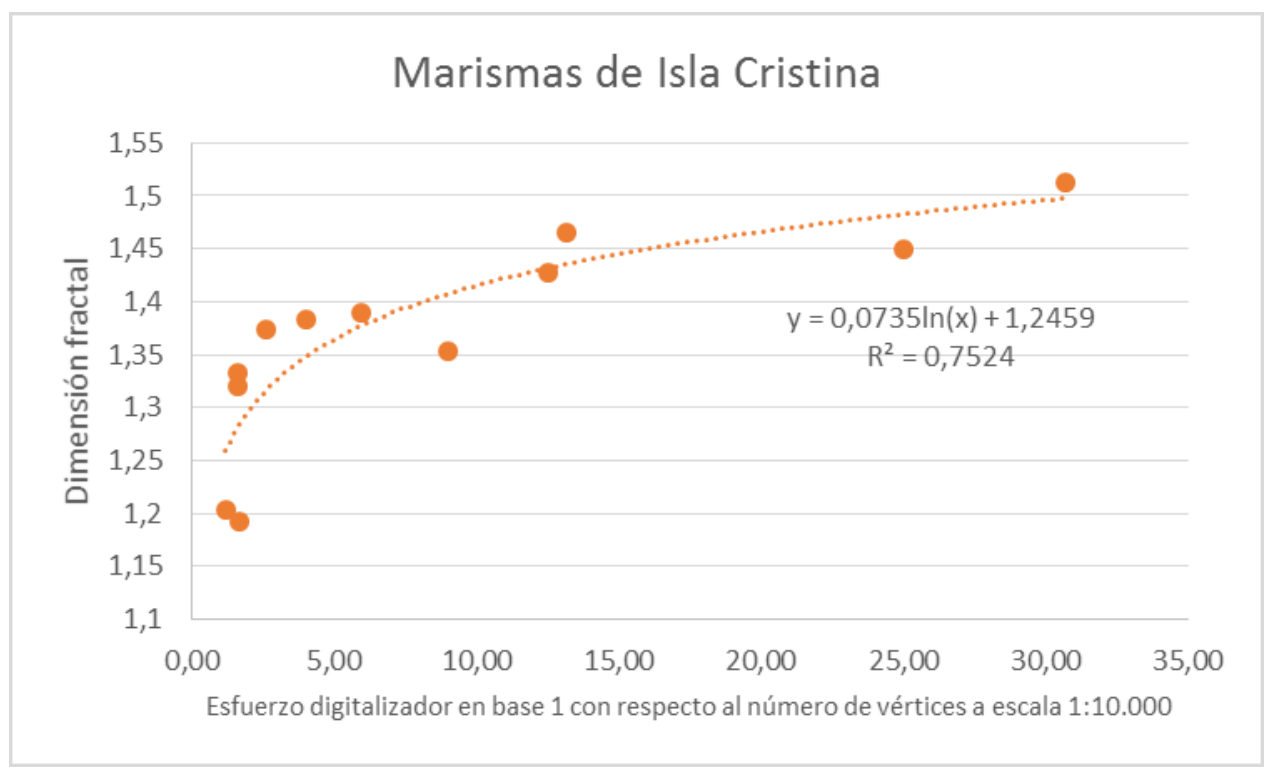

Figura 16. Análisis de regresión mediante modelo logarítmico entre las variables número de vértices (eje $\mathbf{x}$ ) y dimensión fractal (eje y) para el sector de las marismas de Isla Cristina.

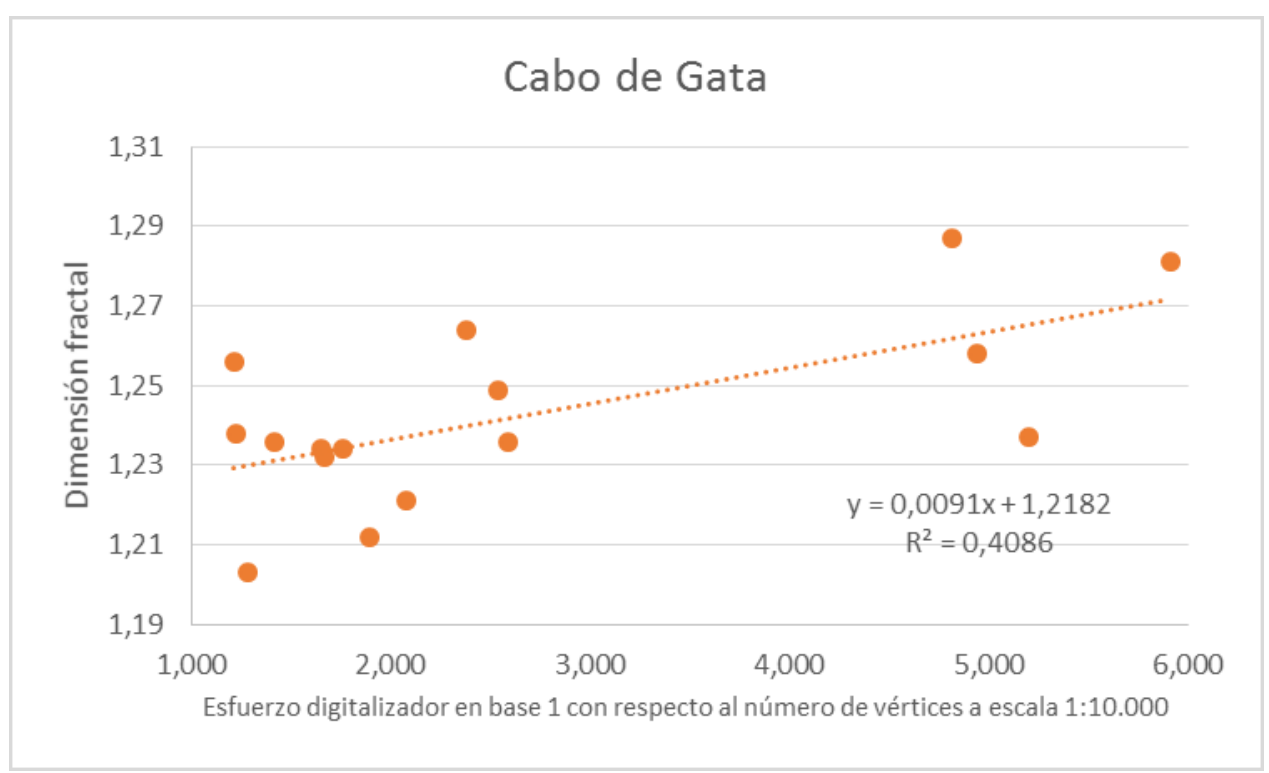

Figura 17. Análisis de regresión mediante modelo logarítmico entre las variables número de vértices (eje $\mathbf{x}$ ) y dimensión fractal (eje y) para el sector del Cabo de Gata. 
Fraile Jurado, P., Fernández Díaz, M. (2018): "Escala, esfuerzo digitalizador y fractalidad en la línea de costa", GeoFocus (Articulos), $n^{\circ}$ 21, p. 253-277. ISSN: 1578-5157 http://dx.doi.org/10.21138/GF.568

\section{Discusión}

Los resultados identifican relaciones entre las variables analizadas: longitud de la línea de costa, número de vértices, y fractalidad. Estas relaciones se han visto reflejadas en la bibliografía parcialmente. Thieler y Danforth (1994) no mencionan la importancia de la escala, centrándose exclusivamente en la importancia de los procesos de preparación de las imágenes, sin considerar ni la escala ni la tipología costera. Los trabajos de Crowell (Crowell et al. 1991; Crowell et al. 1997) han destacado la importancia tanto de mantener una resolución espacial homogénea a lo largo de la costa digitalizada, como de mantener unos criterios homogéneos para el conjunto del objeto digitalizado, concordante con la publicación de Casal et al (2012). Si bien en este trabajo se han mantenido imágenes con resoluciones espaciales homogéneas, y los criterios, tal y como indican Crowell y Casal, son los mismos para todos los tramos digitalizados, una de las principales evidencias obtenidas es que no es preciso emplear la misma escala de digitalización para cada tramo de costa, independientemente de la precisión espacial que se necesite: los tramos rectilíneos o en las infraestructuras costeras (analizadas en los espigones de Málaga) no requieren de una escala de detalle. El empleo de escala de enorme detalle, como el 1:700 aplicado por Casal para la totalidad de la costa gallega, es innecesario, y amplifica los costes de elaboración de una línea de costa. La elección de una escala adecuada de análisis y digitalización parece, pues, ser un punto crítico en cualquier fase de los trabajos de digitalización, independientemente de que hayan sido elaborados por digitalizadores humanos o por sistemas automáticos de digitalización (Gens, 2010). En este sentido, algunos parámetros identificados en este trabajo como la dimensión fractal o la relación entre longitud y esfuerzo digitalizador, podrían ser entendidos como indicadores de la escala óptima de digitalización, tanto manual como automática, tal y como ya ha sido demostrado en cuanto a la dimensión fractal por Sinan et al. (2014).

La fractalidad de la línea de costa se ha empleado anteriormente para la identificación y descripción de diferentes morfologías costeras, como estuarios mesomareales (Ángeles et al., 2004), de tómbolos (Dai et al., 2007), y especialmente para la identificación de áreas erosivas, tanto en morfologías arenosas abiertas (Dai et al., 2004) como en la erosión de marismas (Schwimmer, 2008). El valor identificado por Dai para playas erosivas, de 1,165, coincide con los valores identificados en la playa de Mazagón (Tabla 3 en el anexo), en la que se han identificado tasas de erosión muy altas (Ojeda et al., 2009). Sin embargo, en los trabajos de Ángeles et al. (2004) se alude a un índice fractal de valor 1 para los estuarios de rango mesomareal. Es probable que la diferencia estribe en el método del cálculo del índice fractal, no explicitado en el trabajo de Ángeles et al. (2004) aunque incluso en este supuesto las diferencias son demasiado amplias.

De entre las marismas analizadas, es evidente que las del río Carreras muestran una relación menos intensa entre los diferentes parámetros analizados (Tabla 5), salvo la autocorrelación. Este fenómeno podría quedar explicado por haberse elegido un tramo excesivamente rectilíneo en el sector norte (figura 3), que responde de manera diferente al resto de las marismas del Carreras a los análisis realizados, si bien parecen ser necesarios estudios más detallados para poder explicarlo completamente.

La relación entre fractalidad, escala y línea de costa, asumida por diferentes autores (Sharma \& Byrne, 2010; Dai et al., 2004; Buttenfield, 1989) no se observa de manera constante en los resultados obtenidos en este trabajo, ya que únicamente esignificativa en las marismas y en el 
Fraile Jurado, P., Fernández Díaz, M. (2018): "Escala, esfuerzo digitalizador y fractalidad en la línea de costa", GeoFocus (Articulos), $n^{\circ}$ 21, p. 253-277. ISSN: 1578-5157 http://dx.doi.org/10.21138/GF.568

Cabo de Gata, en el caso de la relación entre dimensión fractal y longitud de línea de costa /figuras 13, 14 y 15), y en Cabo de Gata, Carboneras, marismas de Isla Cristina y del río Piedras para la relación entre dimensión fractal y esfuerzo digitalizador (Tabla 5). No obstante, es preciso señalar que el trabajo de Mandelbroot (1967), que supone el inicio de las matemáticas fractales, no emplea el concepto de escala. Por lo tanto, parece haber existido en diferentes autores una interpretación ambigua de la relación entre fractalidad y escala. Si bien es cierto que la autosimilitud de un objeto complejo (especialmente las marismas y las costas rocosas acantiladas) se incrementa en función de la escala a la que se digitalice, al igual que su longitud, no resulta posible establecer relaciones directas entre fractalidad y longitud, tal y como evidencian las figuras 13, 14 y 15, ni entre fractalidad y esfuerzo digitalizador (tabla 5), si bien es cierto que esta última cuestión no ha sido anteriormente abordada por los autores.

\section{Conclusiones}

A la vista de los resultados obtenidos, se puede que concluir que a la hora de digitalizar, la escala no debe ser constante a lo largo de una línea de costa amplia, puesto que en determinadas costas las ganancias en precisión (longitud de la línea) en comparación con el coste de digitalización, son mínimas. Resulta más apropiado elegir la escala de digitalización en función de las necesidades de precisión del objeto vectorial y de las características de dicho objeto. Por ello, playas e infraestructuras rectilíneas o con pocos ángulos requieren de escalas de poco detalle, mientras que cualquier incremento de la escala para la digitalización de costas rocosas y marismas supone un incremento de la precisión del objeto elaborado. Puesto que el esfuerzo digitalizador depende principalmente de la escala de digitalización y en menor medida, de las características morfológicas del objeto digitalizado, es recomendable realizar una supervisión inicial de los tipos de costa a digitalizar con la intención de decidir las escalas a emplear.

La fractalidad de la línea de costa no resulta un parámetro universalmente explicativo de las características del objeto vectorial digitalizado en la línea de costa. Aunque es un indicador de la complejidad de la costa, su valor varía en función de la escala de digitalización. Este valor no varía de manera homogénea en función ni de la escala ni del tipo de costa.

Aparentemente, existen aproximaciones que pueden permitir prever la longitud y el esfuerzo digitalizar de una línea de costa a partir de digitalizaciones de escaso detalle y poco esfuerzo digitalizador, como las que resultan del uso de una escala 1:10.000. Es posible estimar con notable precisión (a escala 1:1.500) la longitud de ciertas tipologías costeras a partir del número de vértices, dimensión fractal o longitud de la costa a escala 1:10.000. No obstante, los casos de estudio analizados (24 tramos en 7 sectores diferentes) no permiten establecer reglas universales. Será deseable que en el futuro se realicen trabajos con un mayor número de tramos y sectores diferentes, con la intención de elaborar ecuaciones a partir de las cuales poder estimar la longitud de líneas de costa de detalle sin llegar a ser digitalizadas. Este tipo de trabajos podrían llegar a ser de gran utilidad ante la necesidad de trabajos de limpieza, vertidos, etc., en los que es preciso conocer la longitud de la totalidad de la línea de costa. De acuerdo con la bibliografía de referencia, en áreas costeras de alta fractalidad, como marismas y costas rocosas, esta aproximación debería permitir 
Fraile Jurado, P., Fernández Díaz, M. (2018): "Escala, esfuerzo digitalizador y fractalidad en la línea de costa”, GeoFocus (Articulos), $n^{\circ} 21$, p. 253-277. ISSN: 1578-5157 http://dx.doi.org/10.21138/GF.568

alcanzar información relativa a las dimensiones de la escala 1:1, aportando una información de gran valor desde el punto de vista de la gestión.

\section{AGRADECIMIENTOS}

Los autores desean agradecer a Manu López Martín sus comentarios y consejos durante el proceso anterior a la elaboración de este trabajo.

\section{Referencias bibliográficas}

Altinuc, S. O., Keceli, A. S., y Sezer, E. A. (2014). Semi-Automated Shoreline Extraction in Satellite Imagery and Usage of Fractals as Performance Evaluator. International Journal of Computer Theory and Engineering, 6(2), pp. 102-106.

Angeles, G. R., Perillo, G. M., Piccolo, M. C., y Pierini, J. O. (2004): "Fractal analysis of tidal channels in the Bahia Blanca Estuary (Argentina)", Geomorphology, 57(3), pp. 263-274.

Buttenfield, B. P. (1989): "Scale-dependence and self-similarity in cartographic lines", Cartographica: The International Journal for Geographic Information and Geovisualization, 26(1), pp. 79-100.

Boak, E. H., y Turner, I. L. (2005). Shoreline definition and detection: a review. Journal of coastal research, 21 (4), pp. 688-703.

Casal, G., Sánchez-Carnero, N., y Freire, J. (2010): “Generación de una línea de costa digital de Galicia (NW España) a gran escala, utilizando fotointerpretación y segmentación dinámica", Boletín de la Asociación de Geógrafos Españoles, 53, pp. 7-19.

Crowell, M., Leatherman, S. P., y Buckley, M. K. (1991): "Historical shoreline change: error analysis and mapping accuracy", Journal of coastal research, pp. 839-852.

Crowell, M., Douglas, B. C., y Leatherman, S. P. (1997): "On forecasting future US shoreline positions: a test of algorithms", Journal of Coastal Research, pp. 1245-1255.

Crowell, M., Honeycutt, M., y Hatheway, D. (1999): "Coastal erosion hazards study: phase one mapping", Journal of Coastal Research, pp. 10-20.

Dai, Z. J., Li, C. C., y Zhang, Q. L. (2004): "Fractal analysis of shoreline patterns for crenulate-bay beaches, Southern China”, Estuarine, Coastal and Shelf Science, 61(1), pp. 65-71.

Dai, Z. J., Du, J. Z., Li, C. C., y Chen, Z. S. (2007): "The configuration of equilibrium beach profile in South China", Geomorphology, 86(3), pp. 441-454.

Dolan, R., Hayden B.P., May, P., y May S.K. (1980): "The reliability of shoreline change measurements from aerial photographs", Shore and Beach n48 (4), pp. 22-29

Gens, R. (2010). Remote sensing of coastlines: detection, extraction and monitoring. International Journal of Remote Sensing, 31(7), 1819-1836.

INE (1985): Anuario estadístico de España, Instituto Nacional de Estadística, Madrid. 
Fraile Jurado, P., Fernández Díaz, M. (2018): "Escala, esfuerzo digitalizador y fractalidad en la línea de costa”, GeoFocus (Articulos), $n^{\circ}$ 21, p. 253-277. ISSN: 1578-5157 http://dx.doi.org/10.21138/GF.568

Fraile, P. (2011): Análisis de las problemáticas asociadas a la espacialización, evolución y representación de niveles del Mar presentes y futuros en Andalucía, Tesis Doctoral. Universidad de Sevilla.

López-Martín, M. y Fraile-Jurado, P. (2015): “Análisis preliminar del cálculo de la dimensión fractal de diferentes tipos de costa en el litoral andaluz", Geo-temas, 15, pp. 113-116.

Mandelbrot, B. B. (1967): “How long is the coast of Britain”, Science, 156 (3775), pp. 636-638.

Mandelbrot, B. B. (1977): Fractals, John Wiley y Sons, Inc, Hoboken, Nueva Jersey.

Miller, S. M., McWilliams, P. C., y Kerkering, J. C. (1990): "Ambiguities in estimating fractal dimensions of rock fracture surfaces", Proc. 31st US Symp. on Rock Mech, pp. 471-478.

Ojeda, J. (2000): "Métodos para el cálculo de la erosión costera. Revisión, tendencias y propuesta", Boletín de la A.G.E. n³0, pp. 103-118.

Ojeda, J., Álvarez, J. I., Martín, D., y Fraile, P. (2009): "El uso de las TIG para el cálculo del indice de vulnerabilidad costera (CVI) ante una potencial subida del nivel del mar en la costa andaluza (España)", GeoFocus. Revista Internacional de Ciencia y Tecnología de la Información Geográfica, (9), 83-100.

Peltier, W. R., y Tushingham, A. M. (1991): "Influence of glacial isostatic adjustment on tide gauge measurements of secular sea level change", Journal of Geophysical Research: Solid Earth, 96(B4), pp. 6779-6796.

Sharma, P., y Byrne, S. (2010): “Constraints on Titan's topography through fractal analysis of shorelines", Icarus, 209(2), pp. 723-737.

Schwimmer, R. A. (2008): “A temporal geometric analysis of eroding marsh shorelines: can fractal dimensions be related to process?", Journal of Coastal Research, pp. 152-158.

Snow, R. S. (1989): "Fractal sinuosity of stream channels", Pure and applied geophysics, 131(1-2), pp. 99-109.

Terán, M. D., Solé L., Vilá J. (1985) Geografía general de España, Ariel Geografía, Barcelona.

Thieler, E. R., y Danforth, W. W. (1994): "Historical shoreline mapping (I): improving techniques and reducing positioning errors", Journal of Coastal Research, pp. 549-563.

Zhang, K; Huang, W.; Douglas, B.C. y Leatherman, S.P. (2002): "Shoreline position variability and long-term trend analysis", Shore and Beach, no 70 (2), pp. 31-35. 
Fraile Jurado, P., Fernández Díaz, M. (2018): "Escala, esfuerzo digitalizador y fractalidad en la línea de costa", GeoFocus (Artículos), $n^{\circ} 21$, p. 253-277. ISSN: 1578-5157 http://dx.doi.org/10.21138/GF.568

\section{APÉNDICE 1}

Tabla 3. Longitud de los tramos de línea de costa digitalizados a diferentes escalas.

\begin{tabular}{|l|c|c|c|c|c|}
\hline TRAMO & $\begin{array}{c}\text { E: } \\
\mathbf{1 0 . 0 0 0}\end{array}$ & $\mathbf{E : ~ 7 . 5 0 0}$ & $\mathbf{E : ~ 5 . 0 0 0}$ & $\mathbf{E}: \mathbf{2 . 5 0 0}$ & E: 1.500 \\
\hline Cabo de Gata_CE & $2.683,35$ & $2.896,70$ & $2.937,65$ & $2.973,58$ & $3.273,61$ \\
\hline Cabo de Gata_CO & $3.280,14$ & $3.392,40$ & $3.389,75$ & $3.635,62$ & $3.887,94$ \\
\hline Cabo de Gata_E & $5.362,73$ & $5.522,73$ & $5.702,30$ & $5.647,54$ & $6.108,68$ \\
\hline Cabo de Gata_O & $5.201,51$ & $5.254,00$ & $5.367,36$ & $5.406,77$ & $6.058,72$ \\
\hline Carboneras_CE & $1.103,36$ & $1.117,83$ & $1.126,64$ & $1.153,43$ & $1.118,07$ \\
\hline Carboneras_CO & $1.335,04$ & $1.510,54$ & $1.428,73$ & $1.513,90$ & $1.534,10$ \\
\hline Carboneras_E & $2.321,25$ & $2.297,70$ & $2.393,61$ & $2.442,80$ & $2.414,81$ \\
\hline Carboneras_O & $1.023,55$ & $1.102,86$ & $1.106,52$ & $1.200,78$ & $1.226,67$ \\
\hline Málaga_CE & $1.177,75$ & $1.160,94$ & $1.143,80$ & $1.153,20$ & $1.153,76$ \\
\hline Málaga_CO & 993,09 & $1.007,13$ & $1.016,53$ & $1.018,95$ & $1.019,67$ \\
\hline Málaga_E & $1.077,45$ & $1.072,67$ & $1.088,38$ & $1.108,15$ & $1.110,49$ \\
\hline Málaga_O & $1.407,59$ & $1.446,56$ & $1.409,04$ & $1.435,07$ & $1.415,91$ \\
\hline Marismas del Carreras_C & $2.316,01$ & $3.835,81$ & $4.180,24$ & $4.783,55$ & $6.503,03$ \\
\hline Marismas del Carreras_N & $1.619,09$ & $2.668,68$ & $3.168,41$ & $5.703,08$ & $6.552,38$ \\
\hline Marismas del Carreras_S & $3.933,28$ & $5.780,37$ & $8.182,74$ & $10.496,37$ & $12.844,46$ \\
\hline Marismas de Isla Cristina_C & $1.000,34$ & $1.063,61$ & $1.048,29$ & $3.184,40$ & $5.458,32$ \\
\hline Marismas de Isla Cristina_N & $3.108,53$ & $4.450,06$ & $3.884,89$ & $6.380,87$ & $8.433,14$ \\
\hline Marismas de Isla Cristina_S & $4.706,83$ & $7.210,43$ & $8.573,41$ & $14.685,38$ & $21.414,71$ \\
\hline Mazagón_CN & $1.063,45$ & $1.065,36$ & $1.064,25$ & $1.067,19$ & $1.063,42$ \\
\hline Mazagón_CS & $1.046,00$ & $1.034,90$ & $1.037,79$ & $1.036,33$ & $1.042,52$ \\
\hline Mazagón_N & $1.127,66$ & $1.132,47$ & $1.129,92$ & $1.130,49$ & $1.132,18$ \\
\hline Mazagón_S & $1.020,23$ & $1.027,08$ & $1.025,23$ & $1.023,95$ & $1.019,80$ \\
\hline Río Piedras_E & $1.779,21$ & $2.580,77$ & $4.280,99$ & $7.220,15$ & $15.779,87$ \\
\hline Río Piedras_O & $2.239,57$ & $2.981,85$ & $5.121,06$ & $7.573,43$ & $13.763,48$ \\
\hline Río Piedras_S & $3.246,54$ & $3.584,05$ & $5.478,90$ & $6.949,58$ & $9.549,23$ \\
\hline & & & & & \\
\hline
\end{tabular}


Fraile Jurado, P., Fernández Díaz, M. (2018): “Escala, esfuerzo digitalizador y fractalidad en la línea de costa”, GeoFocus (Articulos), $n^{\circ} 21$, p. 253-277. ISSN: 1578-5157 http://dx.doi.org/10.21138/GF.568

Tabla 4. Número de vértices de los tramos digitalizados a diferentes escalas.

\begin{tabular}{|c|c|c|c|c|c|}
\hline TRAMO & $\begin{array}{c}E: \\
10.000\end{array}$ & E: 7.500 & E: 5.000 & E: 2.500 & $\mathrm{E}: \mathbf{1 . 5 0 0}$ \\
\hline Cabo de Gata_CE & 66 & 80 & 116 & 157 & 390 \\
\hline Cabo de Gata_CO & 85 & 104 & 140 & 216 & 420 \\
\hline Cabo de Gata_E & 116 & 149 & 220 & 241 & 603 \\
\hline Cabo de Gata_O & 154 & 218 & 257 & 398 & 741 \\
\hline Carboneras_CE & 28 & 35 & 48 & 76 & 99 \\
\hline Carboneras_CO & 34 & 49 & 64 & 120 & 164 \\
\hline Carboneras_E & 63 & 64 & 113 & 188 & 214 \\
\hline Carboneras_O & 26 & 40 & 54 & 106 & 153 \\
\hline Málaga_CE & 29 & 32 & 38 & 58 & 82 \\
\hline Málaga_CO & 25 & 32 & 33 & 52 & 93 \\
\hline Málaga_E & 25 & 31 & 32 & 49 & 79 \\
\hline Málaga_O & 41 & 54 & 59 & 84 & 142 \\
\hline Marismas del Carreras_C & 49 & 100 & 167 & 261 & 569 \\
\hline Marismas del Carreras_N & 25 & 50 & 85 & 321 & 510 \\
\hline Marismas del Carreras_S & 63 & 142 & 300 & 686 & 1014 \\
\hline Marismas de Isla Cristina_C & 26 & 31 & 44 & 234 & 650 \\
\hline Marismas de Isla Cristina_N & 57 & 91 & 92 & 340 & 715 \\
\hline Marismas de Isla Cristina_S & 71 & 188 & 287 & 933 & 2177 \\
\hline Mazagón_CN & 6 & 10 & 9 & 20 & 21 \\
\hline Mazagón_CS & 9 & 11 & 11 & 15 & 22 \\
\hline Mazagón_N & 10 & 20 & 16 & 24 & 33 \\
\hline Mazagón_S & 7 & 8 & 11 & 15 & 13 \\
\hline Río Piedras_E & 34 & 79 & 186 & 589 & 2272 \\
\hline Río Piedras_O & 39 & 81 & 201 & 557 & 1741 \\
\hline Río Piedras_S & 59 & 84 & 225 & 480 & 1204 \\
\hline
\end{tabular}


Fraile Jurado, P., Fernández Díaz, M. (2018): "Escala, esfuerzo digitalizador y fractalidad en la línea de costa", GeoFocus (Articulos), $n^{\circ} 21$, p. 253-277. ISSN: 1578-5157 http://dx.doi.org/10.21138/GF.568

Tabla 5. Dimensión fractal de cada uno de los tramos digitalizados a diferentes escalas.

\begin{tabular}{|c|c|c|c|c|c|}
\hline TRAMO & $\begin{array}{c}E: \\
10.000\end{array}$ & E: 7.500 & E: 5.000 & E: 2.500 & $\mathrm{E}: 1.500$ \\
\hline Cabo de Gata_CE & 1,247 & 1,256 & 1,234 & 1,264 & 1,281 \\
\hline Cabo de Gata_CO & 1,229 & 1,238 & 1,234 & 1,249 & 1,258 \\
\hline Cabo de Gata_E & 1,211 & 1,203 & 1,212 & 1,221 & 1,237 \\
\hline Cabo de Gata_O & 1,239 & 1,236 & 1,232 & 1,236 & 1,287 \\
\hline Carboneras_CE & 1,243 & 1,249 & 1,245 & 1,25 & 1,261 \\
\hline Carboneras_CO & 1,237 & 1,255 & 1,251 & 1,261 & 1,267 \\
\hline Carboneras_E & 1,176 & 1,172 & 1,183 & 1,244 & 1,246 \\
\hline Carboneras_O & 1,216 & 1,217 & 1,227 & 1,236 & 1,235 \\
\hline Málaga_CE & 1,297 & 1,294 & 1,295 & 1,274 & 1,297 \\
\hline Málaga_CO & 1,265 & 1,269 & 1,271 & 1,273 & 1,269 \\
\hline Málaga_E & 1,271 & 1,29 & 1,264 & 1,269 & 1,274 \\
\hline Málaga_O & 1,329 & 1,333 & 1,331 & 1,331 & 1,335 \\
\hline Marismas del Carreras_C & 1,183 & 1,224 & 1,242 & 1,199 & 1,462 \\
\hline Marismas del Carreras_N & 1,084 & 1,359 & 1,119 & 1,206 & 1,213 \\
\hline Marismas del Carreras_S & 1,204 & 1,399 & 1,228 & 1,268 & 1,303 \\
\hline Marismas de Isla Cristina_C & 1,17 & 1,204 & 1,192 & 1,354 & 1,45 \\
\hline Marismas de Isla Cristina_N & 1,282 & 1,333 & 1,32 & 1,389 & 1,427 \\
\hline Marismas de Isla Cristina_S & 1,314 & 1,374 & 1,383 & 1,465 & 1,512 \\
\hline Mazagón_CN & 1,161 & 1,16 & 1,16 & 1,157 & 1,16 \\
\hline Mazagón_CS & 1,197 & 1,197 & 1,201 & 1,193 & 1,199 \\
\hline Mazagón_N & 1,157 & 1,173 & 1,17 & 1,169 & 1,172 \\
\hline Mazagón_S & 1,166 & 1,163 & 1,164 & 1,165 & 1,163 \\
\hline Río Piedras_E & 1,228 & 1,269 & 1,374 & 1,426 & 1,552 \\
\hline Río Piedras_O & 1,219 & 1,256 & 1,331 & 1,397 & 1,468 \\
\hline Río Piedras_S & 1,349 & 1,334 & 1,359 & 1,39 & 1,431 \\
\hline
\end{tabular}

